DOI 10.4171/JEMS/221

Young-Heon Kim · Robert J. McCann

\title{
Continuity, curvature, and the general covariance of optimal transportation
}

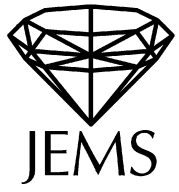

Dedicated to Neil Trudinger, in honour of his 65th birthday. His pioneering works continue to inspire.

Received December 15, 2007 and in revised form June 27, 2008

\begin{abstract}
Let $M$ and $\bar{M}$ be $n$-dimensional manifolds equipped with suitable Borel probability measures $\rho$ and $\bar{\rho}$. For subdomains $M$ and $\bar{M}$ of $\mathbb{R}^{n}$, Ma, Trudinger \& Wang gave sufficient conditions on a transportation cost $c \in C^{4}(M \times \bar{M})$ to guarantee smoothness of the optimal map pushing $\rho$ forward to $\bar{\rho}$; the necessity of these conditions was deduced by Loeper. The present manuscript shows the form of these conditions to be largely dictated by the covariance of the question; it expresses them via non-negativity of the sectional curvature of certain null-planes in a novel but natural pseudo-Riemannian geometry which the cost $c$ induces on the product space $M \times \bar{M}$. We also explore some connections between optimal transportation and spacelike Lagrangian submanifolds in symplectic geometry.

Using the pseudo-Riemannian structure, we extend Ma, Trudinger and Wang's conditions to transportation costs on differentiable manifolds, and provide a direct elementary proof of a maximum principle characterizing it due to Loeper, relaxing his hypotheses even for subdomains $M$ and $\bar{M}$ of $\mathbb{R}^{n}$. This maximum principle plays a key role in Loeper's Hölder continuity theory of optimal maps. Our proof allows his theory to be made logically independent of all earlier works, and sets the stage for extending it to new global settings, such as general submersions and tensor products of the specific Riemannian manifolds he considered.
\end{abstract}

Keywords. Optimal transportation, regularity of optimal maps, Hölder continuity, curvature, covariance, pseudo-Riemannian, semi-Riemannian, para-Kähler, spacelike Lagrangian, lightlike submanifold, signature $(n, n)$, Monge-Kantorovich, measure-preserving homeomorphism

\section{Introduction}

Let $M$ and $\bar{M}$ be Borel subsets of compact separable metric spaces, in which their closures are denoted by $\operatorname{cl} M$ and $\operatorname{cl} \bar{M}$. Suppose $M$ and $\bar{M}$ are equipped with Borel probability measures $\rho$ and $\bar{\rho}$, and let $c: \operatorname{cl}(M \times \bar{M}) \rightarrow \mathbb{R} \cup\{+\infty\}$ be a lower semicontinuous transportation cost defined on the product space. The optimal transportation problem of

Y.-H. Kim: Department of Mathematics, University of Toronto, Toronto, Ontario, Canada M5S 2E4; current address: Department of Mathematics, University of British Columbia, Vancouver, British Columbia, Canada V6T 1Z2; e-mail: yhkim@math.ubc.ca

R. J. McCann: Department of Mathematics, University of Toronto, Toronto, Ontario, Canada M5S 2E4; e-mail: mccann@ math.toronto.edu

Mathematics Subject Classification (2010): 49N60, 35J70, 58E17, $90 \mathrm{~B} 06$ 
Kantorovich [25] is to find the measure $\gamma \geq 0$ on $M \times \bar{M}$ which achieves the infimum

$$
W_{c}(\rho, \bar{\rho}):=\min _{\gamma \in \Gamma(\rho, \bar{\rho})} \int_{M \times \bar{M}} c(x, \bar{x}) d \gamma(x, \bar{x}) .
$$

Here $\Gamma(\rho, \bar{\rho})$ denotes the set of joint probabilities having the same left and right marginals as $\rho \otimes \bar{\rho}$. It is not hard to check that this minimum is attained; any minimizing measure $\gamma \in \Gamma(\rho, \bar{\rho})$ is then called optimal. Each feasible $\gamma \in \Gamma(\rho, \bar{\rho})$ can be thought of as a weighted relation pairing points $x$ distributed like $\rho$ with points $\bar{x}$ distributed like $\bar{\rho}$; optimality implies this pairing also minimizes the average value of the specified cost $c(x, \bar{x})$ of transporting each point $x$ to its destination $\bar{x}$.

The optimal transportation problem of Monge [38] amounts to finding a Borel map $F: M \rightarrow \bar{M}$, and an optimal measure $\gamma$ vanishing outside $\operatorname{Graph}(F):=\{(x, \bar{x}) \in$ $M \times \bar{M} \mid \bar{x}=F(x)\}$. When such a map $F$ exists, it is called an optimal map between $\rho$ and $\bar{\rho}$; in this case, the relation $\gamma$ is single-valued, so that $\rho$-almost every point $x$ has a unique partner $\bar{x}=F(x)$, and optimality can be achieved in (1.1) without subdividing the mass at such points $x$ between different destinations. Although Monge's problem is more subtle to solve than Kantorovich's, when $M$ is a smooth manifold and $\rho$ vanishes on every Lipschitz submanifold of lower dimension, a twist condition ((A1), Definition 2.1 below) on the cost function $c(x, \bar{x})$ guarantees existence and uniqueness of an optimal map $F$, as well as uniqueness of the optimal measure $\gamma$; see Gangbo [21], Levin [30], Carlier [9], and Ma, Trudinger \& Wang [34] for statements of comparable generality, and the works of Brenier, Caffarelli, Gangbo, McCann and others cited in [10], [50]. One can then ask about the smoothness of the optimal map $F: M \rightarrow \bar{M}$.

For Euclidean distance squared $c(x, \bar{x})=|x-\bar{x}|^{2} / 2$, this regularity question was resolved using geometric ideas by Caffarelli [5]-[7], and also by Delanoë in the plane [13] and by Urbas in higher dimensions [44], who formulated it as an oblique boundary value problem and applied the continuity method with a priori estimates. Convexity of $\bar{M} \subset \mathbb{R}^{n}$ necessarily plays a crucial role. Delanoë investigated regularity of optimal transport with respect to Riemannian distance squared on a compact manifold [36], but completed his program only for nearly flat manifolds [14], an improvement on Cordero-Erausquin's result from the torus [11], though his criterion for nearness to flat depends on the measures $\rho$ and $\bar{\rho}$. Under suitable conditions on $\rho$ and $\bar{\rho}$ and domains $M$ and $\bar{M} \subset \mathbb{R}^{n}$, Ma, Trudinger \& Wang [34], [42] developed estimates and a continuity method approach to a class of cost functions $c \in C^{4}(M \times \bar{M})$ which satisfy a mysterious structure condition comparing third and fourth derivatives. Adopting the notation defined in the following section, they express this condition in the form

$$
\sum_{1 \leq i, j \leq n}\left(-c_{i j \bar{k} \bar{l}}+c_{i j \bar{a}} c^{\bar{a} b} c_{\bar{k} \bar{l} b}\right) c^{\bar{k} e} c^{\bar{l} f} p_{i} p_{j} q_{e} q_{f} \geq C|p|^{2}|q|^{2} \quad \text { if } p \perp q,
$$

for some constant $C \geq 0$ and each pair of orthogonal vectors $p, q \in \mathbb{R}^{n}$. Here summation on $\bar{a}, b, e, f, \bar{k}, \bar{l}$ is implicit but the sum on $i, j$ is written explicitly for consistency with our later notation. Loeper [31] confirmed their structure condition to be necessary for continuity of $F$, as well as being sufficient for its smoothness [34], [42], [43]. Loeper furthermore offered a direct argument giving an explicit Hölder exponent for $F$, largely avoiding 
the continuity method, except that he relied on central results of Delanoë, Loeper, Ma, Trudinger and Wang, to establish a kind of maximum principle characterizing (1.2) on certain Riemannian manifolds [14], [34] such as the sphere [16], [32], and on Euclidean domains [42].

Although the tensorial nature of condition (1.2) is in some sense implicit in the works of Ma, Trudinger, Wang and Loeper 1 its full implications have not previously been articulated or explored. In fact, since smoothness of the optimal map is a question whose answer is independent of coordinates on $M$ and $\bar{M}$, it follows that any necessary and sufficient condition for this smoothness should be expressible in terms of coordinate independent quantities such as geodesics and curvature-but geodesics and curvature with respect to what? One of the purposes of this article is to answer this question. We extend the theory of Loeper (and in principle, of Ma, Trudinger \& Wang) to the transportation problem set on a pair of smooth manifolds $M$ and $\bar{M}$, by finding a manifestly covariant expression of Ma, Trudinger \& Wang's structure condition (1.2), as the sectional curvature non-negativity of certain null planes in a pseudo-Riemannian metric on $M \times \bar{M}$ explored here for the first time. In Theorem 3.1 we use this framework to give an elementary and direct geometrical proof of a more general version of the maximum principle which Loeper required to prove Hölder continuity of optimal maps. Our proof is logically independent of the methods and results of Delanoë [14], Delanoë \& Loeper [16], Loeper [31], [32], Ma, Trudinger \& Wang [34], Trudinger \& Wang [42], or their subsequent work [43]. As detailed elsewhere [27], this allows us to make Loeper's proof of Hölder continuity of optimal maps self-contained, including maps minimizing distance squared between mass distributions whose Lebesgue densities satisfy bounds above for $\rho(\cdot)$ and below for $\bar{\rho}(\cdot)$ on the round sphere $M=\bar{M}=\mathbb{S}^{n}$. As a byproduct of our approach, we are able to relax various geometric hypotheses on $M, \bar{M}$ and the cost $c$ required in previous works; a few of these relaxations were also obtained simultaneously and independently by Trudinger \& Wang using a different approach [43], which we learned of while this paper was still in a preliminary form [28].

An important feature of our theory is in its geometric and global nature. In combination with our results from [29], this allows us to extend the conclusions of the key Theorem 3.1 to new global settings, including for example the Riemannian distance squared on the product $M=\bar{M}=\mathbb{S}^{n_{1}} \times \cdots \times \mathbb{S}^{n_{k}} \times \mathbb{R}^{l}$ of round spheres, or Riemannian submersions thereof. This is a genuine advantage of our work over other approaches [42], [43], [31], [32] (see Example 3.9 and Remark 3.10). It has been further exploited by several subsequent authors, as we note in Remark 4.13

\footnotetext{
1 Tensoriality was observed en passant in [40], while an assertion and proof of this fact has been included in the revised version of [31], which was communicated to Villani and recorded by him independently of the present work; see notes to Chapter 12 in $\mid 50]$. Loeper was motivated to call the expression appearing in (1.2) a cost-sectional curvature by his discovery of its coincidence with ordinary Riemannian sectional curvature along the diagonal in the special case $\left(M \times M, c=d^{2} / 2\right)$ of Example 3.6 However, it is only in the present article that the same expression was revealed quite generally to be a pseudo-Riemannian sectional curvature with respect to a metric induced by the cost function. In hindsight, this could have been anticipated from the fact that curvatures arise whenever diffeomorphism invariance is present, as in Einstein's theory of gravity [51].
} 
Since terms like $c$-convex, $c$-subdifferential, and notations like $\partial^{c} u$ are used inconsistently through the literature, and because we wish to recast the entire conceptual framework into a pseudo-Riemannian setting, we often depart from the notation and terminology developed by Ma, Trudinger, Wang and Loeper. Instead, we have tried to make the mathematics accessible to a different readership, by choosing language intended to convey the geometrical structure of the problem and its connection to classical concepts in differential geometry not overly specialized to optimal transportation or fully nonlinear differential equations. This approach has the advantage of inspiring certain intuitions about the problem which are quite distinct from those manifested in the previous approaches, and has a structure somewhat reminiscent of symplectic or complex geometry. Although we were initially surprised to discover that the intrinsic geometry of optimal transportation is pseudo-Riemannian, with hindsight we explain why this must be the case, and make some connections to symplectic geometry and Lagrangian submanifolds in the concluding remarks and appendix.

The outline of this paper is as follows. In the next section we introduce the pseudoRiemannian framework and use it to adapt the relevant concepts and structures from Ma, Trudinger \& Wang's work on Euclidean domains to manifolds whose only geometric structure arises from a cost function $c: M \times \bar{M} \rightarrow \mathbb{R} \cup\{+\infty\}$. Since Morse theory prevents a smooth cost from satisfying the desired hypothesis (A1) on a compact manifold, we deal from the outset with functions which may fail to be smooth on a small set-such as the cut locus in the Riemannian setting [36], [32] (see Example 3.6, or the diagonal in the reflector antenna problem [24], [53], [8] (see Example 3.5). This is followed by Section 3, where we motivate and state the main theorem proved here: a version of Loeper's geometric characterization of (1.2) which we call the double-mountain above sliding-mountain maximum principle. In the same section we illustrate how this theorem and the pseudo-Riemannian framework shed new light on a series of variations of familiar examples from Ma, Trudinger, Wang and Loeper, including those discussed above, and new ones formed from these by quotients and tensor products of, e.g., round spheres of different sizes, in Example 3.9 Section 4 contains the proofs which relate our definitions to theirs and establish the main theorems. A level set argument is required to handle the more delicate case in which the positivity in 1.2 is not strict. The last section offers some perspective on these results and their connection to optimal transportation, and is followed by an appendix giving a differential-geometric characterization of optimality among differentiable maps. In a separate work which originated as a series of appendices to the present manuscript, we give a complete account of Loeper's theory of Hölder continuity of optimal mappings, illustrating how our main result makes this theory self-contained, and simplifying the argument at a few points [27]: in particular, we give a unified treatment of the Riemannian sphere and reflector antenna problems, using the fact that the mapping is continuous in the former to deduce the fact that it avoids the cut locus [16], instead of the other way around. 


\section{Pseudo-Riemannian framework}

Fix manifolds $M$ and $\bar{M}$ which, if not compact, are continuously embedded in separable metrizable spaces where their closures $\operatorname{cl} M$ and $\operatorname{cl} \bar{M}$ are compact. Equip $M$ and $\bar{M}$ with Borel probability measures $\rho$ and $\bar{\rho}$, and a lower semicontinuous cost function $c: \operatorname{cl}(M \times$ $\bar{M}) \rightarrow \mathbb{R} \cup\{+\infty\}$, and a subdomain $N \subset M \times \bar{M}$ of the product manifold. Visualize the relation $N$ as a multivalued map and denote its inverse by $\breve{N}:=\{(\bar{x}, x) \mid(x, \bar{x}) \in N\}$. We call $\bar{N}(x):=\{\bar{x} \in \bar{M} \mid(x, \bar{x}) \in N\}$ the set of destinations visible from $x$, and $N(\bar{x}):=\{x \in M \mid(x, \bar{x}) \in N\}$ the set of sources visible from $\bar{x}$. We define the reflected cost $\check{c}(\bar{x}, x):=c(x, \bar{x})$ on $\bar{M} \times M$. In local coordinates $x^{1}, \ldots, x^{n}$ on $M$ and $x^{\overline{1}}, \ldots, x^{\bar{n}}$ on $\bar{M}$, we use the notation such as $c_{i}=\partial c / \partial x^{i}$ and $c_{\bar{i}}=\partial c / \partial x^{\bar{i}}$ to denote the partial derivatives $D c=\left(c_{1}, \ldots, c_{n}\right)$ and $\bar{D} c=\left(c_{\overline{1}}, \ldots, c_{\bar{n}}\right)$ of the cost, and $c_{i \bar{j}}=\partial^{2} c / \partial x^{\bar{j}} \partial x^{i}$ to denote the mixed partial derivatives, which commute with each other and form the coefficients in the $n \times n$ matrix $\bar{D} D c$. When $c_{i \bar{j}}$ is invertible its inverse matrix will be denoted by $c^{\bar{j} k}$. The same notation is used for tensor indices, with repeated indices being summed from 1 to $n$ (or $n+1$ to $2 n$ in the case of barred indices), unless otherwise noted.

Let $T_{x} M$ and $T_{x}^{*} M$ denote the tangent and cotangent spaces to $M$ at $x$. Since the manifold $N \subset M \times \bar{M}$ has a product structure, its tangent and cotangent spaces split canonically: $T_{(x, \bar{x})} N=T_{x} M \oplus T_{\bar{x}} \bar{M}$ and $T_{(x, \bar{x})}^{*} N=T_{x}^{*} M \oplus T_{\bar{x}}^{*} \bar{M}$. For $c(x, \bar{x})$ sufficiently smooth, this canonical splitting of the one-form $d c$ will be denoted by $d c=D c \oplus \bar{D} c$. Similarly, although the Hessian of $c$ is not uniquely defined until a metric has been selected on $N$, the cross partial derivatives $\bar{D} D c$ at $(x, \bar{x}) \in N$ define an unambiguous linear map from vectors at $\bar{x}$ to covectors at $x$; the adjoint $(\bar{D} D c)^{\dagger}=D \bar{D} c$ of this map takes $T_{x} M$ to $T_{\bar{x}}^{*} \bar{M}$. Thus

$$
h:=\frac{1}{2}\left(\begin{array}{cc}
0 & -\bar{D} D c \\
-D \bar{D} c & 0
\end{array}\right)
$$

gives a symmetric bilinear form on the tangent space $T_{(x, \bar{x})} N$ to the product. Let us now adapt the assumptions of Ma, Trudinger \& Wang [34], [42] to manifolds:

(A0) (Smoothness) $c \in C^{4}(N)$.

Definition 2.1 (Twist condition). A cost $c \in C^{1}(N)$ is called twisted if

(A1) for all $x \in M$ the map $\bar{x} \mapsto-D c(x, \bar{x})$ from $\bar{N}(x) \subset \bar{M}$ to $T_{x}^{*} M$ is injective.

If $c$ is twisted on $N \subset M \times \bar{M}$ and also $\check{c}(\bar{x}, x)=c(x, \bar{x})$ is twisted on $\breve{N}=\{(\bar{x}, x) \mid$ $(x, \bar{x}) \in N\}$ we say $c$ is bi-twisted.

Definition 2.2 (Non-degeneracy). A cost $c \in C^{2}(N)$ is non-degenerate if

(A2) for all $(x, \bar{x}) \in N$ the linear map $\bar{D} D c: T_{\bar{x}} \bar{M} \rightarrow T_{x}^{*} M$ is bijective.

Though (A1) will not really be needed hereafter, for suitable probability measures $\rho$ and $\bar{\rho}$ on $M$ and $\bar{M}$ the twist condition alone is enough to guarantee the Kantorovich infimum 1.1 is uniquely attained, as well as existence of an optimal map $F: M \rightarrow \bar{M}$ [21], 
[30], [34], as reviewed in [50], [10]. It implies the dimension of $\bar{M}$ cannot exceed that of $M$, while (A2) forces these two dimensions to coincide. The non-degeneracy condition (A2) ensures the map $\bar{x} \mapsto-D c(x, \bar{x})$ acts as a local diffeomorphism from $\bar{N}(x) \subset \bar{M}$ to a subset of $T_{x}^{*} M$ (which becomes global if the cost is twisted, in which case its inverse is called the cost-exponential, [31], Definition 4.3 below), and that $h(\cdot, \cdot)$ defined by (2.1) is a non-degenerate symmetric bilinear form on $T_{(x, \bar{x})} N$. Although $h$ is not positive definite, it defines a pseudo-Riemannian metric on $N$, which might also be denoted by $d \ell^{2}=-c_{i}{ }_{j} d x^{i} d x^{\bar{j}}$. The signature of this metric is zero, since in any choice of coordinates on $M$ and $\bar{M}$, the eigenvalues of $h$ come in $\pm \lambda$ pairs due to the structure 2.1 ; the corresponding eigenvectors are $p \oplus \bar{p}$ and $(-p) \oplus \bar{p}$ in $T_{(x, \bar{x})} N=T_{x} M \oplus T_{\bar{x}} M$. Non-degeneracy ensures there are no zero eigenvalues. A vector $p \oplus \bar{p}$ is called null if $h(p \oplus \bar{p}, p \oplus \bar{p})=0$. A submanifold $\Sigma \subset N$ is called $n u l l$ if all its tangent vectors are null vectors, and totally geodesic if each geodesic curve tangent to $\Sigma$ at a point is contained in $\Sigma$ locally. The submanifolds $\{x\} \times \bar{N}(x)$ and $N(\bar{x}) \times\{\bar{x}\}$ are examples of null submanifolds in this geometry, and will turn out to be totally geodesic. Assuming $c \in C^{4}(N)$, we can use the Riemann curvature tensor $R_{i^{\prime} j^{\prime} k^{\prime} l^{\prime}}$ induced by $h$ on $N$ to define the sectional curvature of a two-plane $P \wedge Q$ at $(x, \bar{x}) \in N$ :

$$
\sec _{(x, \bar{x})} P \wedge Q=\sec _{(x, \bar{x})}^{(N, h)} P \wedge Q=\sum_{i^{\prime}=1}^{2 n} \sum_{j^{\prime}=1}^{2 n} \sum_{k^{\prime}=1}^{2 n} \sum_{l^{\prime}=1}^{2 n} R_{i^{\prime} j^{\prime} k^{\prime} l^{\prime}} P^{i^{\prime}} Q^{j^{\prime}} P^{k^{\prime}} Q^{l^{\prime}} .
$$

In this geometrical framework, we reformulate the mysterious structure condition (1.2) of Ma, Trudinger \& Wang [34], [42] from the Euclidean setting, which was necessary for continuity of optimal maps [31] and sufficient for regularity [34], [42]. The reader is able to recover their condition from ours by computing the Riemann curvature tensor 4.2). Note that we do not normalize our sectional curvature definition $(2.2)$ by dividing by the customary quantity $h(P, P) h(Q, Q)-h(P, Q)^{2}$, since this quantity vanishes in the case of most interest to us, namely $P=p \oplus 0$ orthogonal to $Q=0 \oplus \bar{p}$, which means $p \oplus \bar{p}$ is null.

Definition 2.3 (Regular costs and cross-curvature). A cost $c \in C^{4}(N)$ is weakly regular on $N$ if it is non-degenerate and for every $(x, \bar{x}) \in N$,

(A3w) $\sec _{(x, \bar{x})}(p \oplus 0) \wedge(0 \oplus \bar{p}) \geq 0$ for all null vectors $p \oplus \bar{p} \in T_{(x, \bar{x})} N$.

A weakly regular cost function is strictly regular on $N$ if equality in (A3w) implies $p=0$ or $\bar{p}=0$, in which case we say (A3s) holds on $N$. We refer to the quantity appearing in 2.3 as the cross-curvature, and say a weakly regular cost - and the pseudo-metric 2.1 it induces on $\mathrm{N}$-are non-negatively cross-curved if

$$
\sec _{(x, \bar{x})}(p \oplus 0) \wedge(0 \oplus \bar{p}) \geq 0
$$

for all $(x, \bar{x}) \in N$ and $p \oplus \bar{p} \in T_{(x, \bar{x})} N$, not necessarily null. The cost $c$ and geometry $(N, h)$ are said to be positively cross-curved if, in addition, equality in 2.3) implies $p=0$ or $\bar{p}=0$. 
If cl $M \subset \subset M^{\prime}$ and $\mathrm{cl} \bar{M} \subset \subset \bar{M}^{\prime}$ are contained in larger manifolds and (A0), (A2) and (A3s/w) all hold on some neighbourhood $N^{\prime} \subset M^{\prime} \times \bar{M}^{\prime}$ containing $N \subset \subset N^{\prime}$ compactly, we say $c$ is strictly/weakly regular on $\mathrm{cl} N$. If, in addition (A1) holds on $N^{\prime}$, we say $c$ is twisted on $\mathrm{cl} N$.

The nullity condition on $p \oplus \bar{p}$ distinguishes weak regularity of the cost from nonnegative cross-curvature: this distinction is important in Examples 3.5 and 3.9 among others; see also Trudinger \& Wang [42]. Non-negative cross-curvature is in turn a weaker condition than $\sec ^{(N, h)} \geq 0$, which means $\sec _{(x, \bar{x})}(p \oplus \bar{q}) \wedge(q \oplus \bar{p}) \geq 0$ for all $(x, \bar{x}) \in N$ and $p \oplus \bar{q}, q \oplus \bar{p} \in T_{(x, \bar{x})} N$. As a consequence of Lemma 4.1, and due to the special form of the pseudo-metric, $\sec ^{(N, h)} \geq 0$ is equivalent to requiring non-negativity of the cross-curvature operator as a quadratic form on the vector space $T_{x} M \wedge T_{\bar{x}} \bar{M}$, i.e.,

$$
R_{i \bar{j} k \bar{l}}\left(p^{i} p^{\bar{j}}-q^{i} q^{\bar{j}}\right)\left(p^{k} p^{\bar{l}}-q^{k} q^{\bar{l}}\right) \geq 0 .
$$

Example 2.4 (Strictly convex boundaries). Let $\Omega \subset \mathbb{R}^{n+1}$ and $\Lambda \subset \mathbb{R}^{n+1}$ be bounded convex domains with $C^{2}$-smooth boundaries. Set $M=\partial \Omega, \bar{M}=\partial \Lambda$, and $c(x, \bar{x})=$ $|x-\bar{x}|^{2} / 2$. We claim the pseudo-metric 2.1 is non-degenerate and that $\sec ^{(N, h)} \geq 0$ on $N:=\left\{(x, \bar{x}) \in \partial \Omega \times \partial \Lambda \mid \hat{n}_{\Omega}(x) \cdot \hat{n}_{\Lambda}(\bar{x})>0\right\}$, where $\hat{n}_{\Omega}(x)$ denotes the outer normal to $\Omega$ at $x$. Indeed, fixing $(x, \bar{x}) \in N$, parameterize $M$ near $x$ as a graph $X \in \mathbb{R}^{n} \mapsto$ $(X, f(X)) \in \partial \Omega$ over the hyperplane orthogonal to $\hat{n}_{\Omega}(x)+\hat{n}_{\Lambda}(\bar{x})$, and $\bar{M}$ near $\bar{x}$ by a convex graph $\bar{X} \in \mathbb{R}^{n} \mapsto(\bar{X}, g(\bar{X}))$ over the same hyperplane. This choice of hyperplane guarantees $|\nabla f(X)|<1$ and $|\nabla g(\bar{X})|<1$ nearby, so in the canonical coordinates and inner product on $\mathbb{R}^{n}$, (A2)-(A3w) follow from a computation of Ma, Trudinger \& Wang [34] which yields the cross-curvature

$$
\sec _{(x, \bar{x})}^{(N, h)}(p \oplus 0) \wedge(0 \oplus \bar{p})=\left(p^{i} f_{i k} p^{k}\right)\left(p^{\bar{j}} g_{\bar{j} \bar{l}} p^{\bar{l}}\right) /(2+2 \nabla f \cdot \nabla g) \geq 0 .
$$

In fact, we can also deduce the stronger conclusion $\sec ^{(N, h)} \geq 0$ as in 2.4):

$$
\begin{aligned}
(2+2 \nabla f & \cdot \nabla g) \sec _{(x, \bar{x})}^{(N, h)}(p \oplus \bar{q}) \wedge(q \oplus \bar{p}) \\
& =\left\langle p D^{2} f p\right\rangle\left\langle\bar{p} D^{2} g \bar{p}\right\rangle+\left\langle q D^{2} f q\right\rangle\left\langle\bar{q} D^{2} g \bar{q}\right\rangle-2\left\langle p D^{2} f q\right\rangle\left\langle\bar{p} D^{2} g \bar{q}\right\rangle \\
& \geq\left(\sqrt{\left\langle p D^{2} f p\right\rangle\left\langle\bar{p} D^{2} g \bar{p}\right\rangle}-\sqrt{\left\langle q D^{2} f q\right\rangle\left\langle\bar{q} D^{2} g \bar{q}\right\rangle}\right)^{2} .
\end{aligned}
$$

Noting $\hat{n}_{\Omega}((X, f(X)))=(\nabla f(X),-1)$, Ma, Trudinger \& Wang's computation shows nondegeneracy (A2) fails at the boundary of $N$ where $\hat{n}_{\Omega}(x) \cdot \hat{n}_{\Lambda}(\bar{x})=0$ implies the denominator of 2.5] is zero. Gangbo \& McCann [23] showed the cost is twisted on $N$ provided $\Lambda$ is strictly convex, but cannot be twisted on any larger domain in $M \times \bar{M}$. If both $\Omega$ and $\Lambda$ are 2-uniformly convex, meaning that the Hessians $D^{2} f$ and $D^{2} g$ are positive definite, the conditions for equality in 2.6$)$ show the sectional curvature of $(N, h)$ to be positive. The resulting strict regularity (A3s) underlies Gangbo \& McCann's proof of continuity for each of the multiple mappings which — due to the absence of twisting (A1) - are required to support the unique optimizer $\gamma \in \Gamma(\rho, \bar{\rho})$ in this geometry. Here the probability measures $\rho$ and $\bar{\rho}$ are assumed to be mutually absolutely continuous with respect to 
surface measure on $\Omega$ and $\Lambda$, both having densities bounded away from zero and infinity. For contrast, observe in this case that the same computations show that although nondegeneracy (A2) also holds on the set $N_{-}:=\left\{(x, \bar{x}) \in \partial \Omega \times \partial \Lambda \mid \hat{n}_{\Omega}(x) \cdot \hat{n}_{\Lambda}(\bar{x})<0\right\}$, this time both surfaces can be expressed locally as graphs over $\hat{n}_{\Omega}(x)-\hat{n}_{\Lambda}(\bar{x})$ but (A3w) fails at each point $(x, \bar{x}) \in N_{-}$: indeed, the cross-curvatures of $N_{-}$are all negative because $D^{2} f>0>D^{2} g$ have opposite signs.

Let us now exploit the geodesic structure which the pseudo-metric $h$ induces on $N \subset$ $M \times \bar{M}$ to recover Ma, Trudinger \& Wang's notions concerning $c$-convex domains [34] in our setting.

Definition 2.5 (Notions of convexity). A subset $W \subset N \subset M \times \bar{M}$ is geodesically convex if each pair of points in $W$ is linked by a curve in $W$ satisfying the geodesic equation on $(N, h)$. This definition is extended to subsets $W \subset \operatorname{cl} N$ by allowing geodesics in $N$ which have endpoints on $\partial N$. We say $\bar{\Omega} \subset \mathrm{cl} \bar{M}$ appears convex from $x \in M$ if $\{x\} \times \bar{\Omega}$ is geodesically convex and $\bar{\Omega} \subset \operatorname{cl} \bar{N}(x)$. We say $W \subset M \times \bar{M}$ is vertically convex if $\bar{W}(x):=\{\bar{x} \in \bar{M} \mid(x, \bar{x}) \in W\}$ appears convex from $x$ for each $x \in M$. We say $\Omega \subset \operatorname{cl} M$ appears convex from $\bar{x} \in \bar{M}$ if $\Omega \times\{\bar{x}\}$ is geodesically convex and $\Omega \subset \operatorname{cl} N(\bar{x})$. We say $W \subset M \times \bar{M}$ is horizontally convex if $W(\bar{x}):=\{x \in M \mid(x, \bar{x}) \in W\}$ appears convex from $\bar{x}$ for each $\bar{x} \in \bar{M}$. If $W$ is both vertically and horizontally convex, we say it is bi-convex.

For a non-degenerate twisted cost (A0)-(A2), Lemma 4.4 shows $\bar{\Omega} \subset \bar{N}(x)$ appears convex from $x$ if and only if $D c(x, \bar{\Omega})$ is convex in $T_{x}^{*} M$; similarly for a bi-twisted cost $\Omega \subset N(\bar{x})$ appears convex from $\bar{x}$ if and only if $\bar{D} c(\Omega, \bar{x})$ is convex in $T_{\bar{x}}^{*} \bar{M}$. This leads immediately to notions of apparent strict convexity, and apparent uniform convexity for such sets, and shows our definition of apparent convexity is simply an adaptation to manifolds of the $c$-convexity and $\check{c}$-convexity of Ma, Trudinger \& Wang [34]: $\Omega$ is $\check{c}$ convex in their language with respect to $\bar{x}$ if it appears convex from $\bar{x} ; \bar{\Omega}$ is $c$-convex with respect $x$ if it appears convex from $x$; and $\Omega$ and $\bar{\Omega}$ are $\check{c}$ - and $c$-convex with respect to each other if $N=\Omega \times \bar{\Omega} \subset \mathbb{R}^{2 n}$ is bi-convex, meaning $D c(x, \bar{N}(x)) \subset T_{x}^{*} M$ and $\bar{D} c(N(\bar{x}), \bar{x}) \subset T_{\bar{x}}^{*} \bar{M}$ are convex domains for each $(x, \bar{x}) \in N$.

Remark 2.6. In dimension $n=1$, strict regularity (A3s) follows vacuously from nondegeneracy (A2), since $p \oplus \bar{p}$ null implies $p=0$ or $\bar{p}=0$. For this reason we generally discuss $n \geq 2$ hereafter. Note, however, that for $n=1$ and $c \in C^{4}(N)$ non-degenerately twisted, $N$ is bi-convex if and only if $N(\bar{x})$ and $\bar{N}(x)$ are homeomorphic to intervals. In local coordinates $x^{1}$ and $\bar{x}^{1}$ on $N$, non-degeneracy implies $c_{1 \overline{1}}=\mp e^{ \pm \lambda\left(x^{1}, \bar{x}^{1}\right)}$. Comparing $-c_{11 \overline{1} \overline{1}}+c_{11 \overline{1}} c^{\overline{1} 1} c_{1 \overline{1} \overline{1}}=\lambda_{1 \overline{1}}\left|c_{1 \overline{1}}\right|$ with 4.2 shows

$$
c\left(x^{1}, \bar{x}^{1}\right)=\mp \int_{x_{0}}^{x^{1}} \int_{\bar{x}_{0}}^{\bar{x}^{1}} e^{ \pm \lambda(s, t)} d s d t
$$

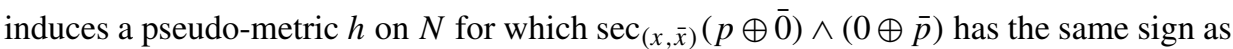
$\partial^{2} \lambda / \partial x^{1} \partial \bar{x}^{1}$ whenever $p \neq 0 \neq \bar{p}$. If $(N, h)$ is connected its cross-curvature will therefore have a definite sign if $\lambda(x, \bar{x})$ is non-degenerate, and a semidefinite sign if $\lambda(x, \bar{x})$ 
is twisted. Moreover, the sign of the cross-curvature, sectional curvature, and curvature operator all coincide on a product of one-dimensional manifolds, although this would not necessarily be true on a product of surfaces or higher-dimensional manifolds.

Definition 2.7 ( $c$-contact set). Given $\Omega \subset \operatorname{cl} M, u: \Omega \rightarrow \mathbb{R} \cup\{+\infty\}$, and $c: \operatorname{cl}(M \times$ $\bar{M}) \rightarrow \mathbb{R} \cup\{+\infty\}$, we define $\operatorname{Dom} c:=\{(x, \bar{x}) \in \operatorname{cl}(M \times \bar{M}) \mid c(x, \bar{x})<\infty\}$, the $c$-contact set $\partial_{\Omega}^{c} u(x):=\left\{\bar{x} \in \operatorname{cl} \bar{M} \mid(x, \bar{x}) \in \partial_{\Omega}^{c} u\right\}$, and $\partial^{c} u=\partial_{\mathrm{cl}}^{c} M^{u}$, where

$$
\partial_{\Omega}^{c} u:=\{(x, \bar{x}) \in \operatorname{Dom} c \mid u(y)+c(y, \bar{x}) \geq u(x)+c(x, \bar{x}) \text { for all } y \in \Omega\} .
$$

We define Dom $\partial_{\Omega}^{c} u:=\left\{x \in \Omega \mid \partial_{\Omega}^{c} u(x) \neq \emptyset\right\}$ and Dom $\partial^{c} u:=\operatorname{Dom} \partial_{\mathrm{cl} M}^{c} u$.

\section{Main results and examples}

A basic result of Loeper [31] states that a cost satisfying (A0)-(A2) on a bi-convex domain $N=M \times \bar{M} \subset \mathbb{R}^{n} \times \mathbb{R}^{n}$ is weakly regular (A3w) if and only if $\partial^{c} u(x)$ appears convex from $x$ for each function $u: M \rightarrow \mathbb{R} \cup\{+\infty\}$ and each $x \in M$. His necessity argument is elementary and direct, but for sufficiency he appeals to a result of Trudinger \& Wang which required $c$-boundedness of the domains $M$ and $\bar{M}$ in the original version of [42]. The same authors gave another proof of sufficiency for strictly regular costs in [43], and removed the $c$-boundedness restriction in the subsequent revision of [42]. Our main result is a direct proof of this sufficiency, found independently but simultaneously with [43], under even weaker conditions on the cost function and domain geometry. In particular, the manifolds $M$ and $\bar{M}$ in Theorem 3.1 need not be equipped with global coordinate charts or Riemannian metrics, the open set $N \subset M \times \bar{M}$ need not have a product structure, and the weakly regular cost need neither be twisted nor strictly regular. This freedom proves useful in Examples 2.4 and 3.9 and Remark 3.10

Theorem 3.1 (Weak regularity connects $c$-contact sets). Use a cost $c: \operatorname{cl}(M \times \bar{M}) \rightarrow$ $\mathbb{R} \cup\{+\infty\}$ with non-degenerate restriction $c \in C^{4}(N)$ to define a pseudo-metric 2.1 on a horizontally convex domain $N \subset M \times \bar{M}$. Fix $\Omega \subset \operatorname{cl} M, x \in M$, and a set $\bar{\Omega} \subset \operatorname{cl} N(x)$ which appears convex from $x$. Suppose $\bigcap_{0 \leq t \leq 1} N(\bar{x}(t))$ is dense in $\Omega$ for each geodesic $t \in[0,1] \mapsto(x, \bar{x}(t)) \in\{x\} \times \bar{\Omega}$, and $c: \operatorname{cl}(N) \rightarrow \mathbb{R} \cup\{+\infty\}$ is continuous. If $c$ is weakly regular (A3w) on $N$, then $\bar{\Omega} \cap \partial_{\Omega}^{c} u(x)$ is connected (and in fact appears convex from $x$ ) for each $u: \Omega \rightarrow \mathbb{R} \cup\{+\infty\}$ with $x \in \operatorname{Dom} u$.

To motivate the proof of this theorem and its relevance to the economics of transportation, consider the optimal division of mass $\rho(\cdot)$ between two target points $\bar{y}, \bar{z} \in \bar{M}$ in ratio $(1-\epsilon) / \epsilon$. This corresponds to the minimization 1.11 with $\bar{\rho}(\cdot)=(1-\epsilon) \delta_{\bar{y}}(\cdot)+\epsilon \delta_{\bar{z}}(\cdot)$. If $c(x, \bar{x})$ is the cost of transporting each commodity unit from $x$ to $\bar{x}$, a price differential $\lambda$ between the fair market value of the same commodity at $\bar{z}$ and $\bar{y}$ will tend to balance demand $\epsilon$ and $1-\epsilon$ with supply $\rho(\cdot)$, given the relative proximity of $\bar{z}$ and $\bar{y}$ to the producers $\rho(\cdot)$ distributed throughout $M$. Here proximity is measured by transportation cost. Since each producer will sell his commodity at $\bar{z}$ or $\bar{y}$, depending on which of these 
two options maximizes his profit, the economic equilibrium and optimal solution will be given, e.g. as in [22], by finding the largest $\lambda \in \mathbb{R}$ such that

$$
u(x)=\max \{\lambda-c(x, \bar{y}),-c(x, \bar{z})\} \quad \text { yields } \quad \epsilon \leq \rho[\{x \in M \mid u(x)=-c(x, \bar{z})\}] .
$$

Producers in the region $\{x \in M \mid \lambda-c(x, \bar{y})>-c(x, \bar{z})\}$ will choose to sell their commodities at $\bar{y}$, while producers in the region where the opposite inequality holds will choose to sell their commodities at $\bar{z}$; points $x_{0} \in M$ on the hypersurface $c\left(x_{0}, \bar{y}\right)-$ $c\left(x_{0}, \bar{z}\right)=\lambda$ of equality are indifferent between the two possible sale destinations $\bar{y}$ and $\bar{z}$. We call this hypersurface the valley of indifference, since it corresponds to a crease in the graph of the function $u$. Loeper's observation is that for optimal mappings to be continuous, each point $x_{0}$ in the valley of indifference between $\bar{y}$ and $\bar{z}$ must also be indifferent to a continuous path of points $\bar{x}(t)$ linking $\bar{y}=\bar{x}(0)$ to $\bar{z}=\bar{x}(1)$; otherwise, he constructs a measure $\rho$ concentrated near $x_{0}$ for which the optimal map to a mollified version of $\bar{\rho}$ exhibits a discontinuous jump, since arbitrarily close producers will choose to supply very different consumers. Indifference means one can choose $\lambda(t)$ such that

$$
u(x) \geq \max _{0<t<1}(\lambda(t)-c(x, \bar{x}(t)))
$$

for all $x \in M$, with equality at $x_{0}$ for each $t \in[0,1]$. When the path connecting $\bar{y}$ to $\bar{z}$ exists, this equality forces $\lambda(t)=c\left(x_{0}, \bar{x}(t)\right)-c\left(x_{0}, \bar{z}\right)$; it also forces the path $t \in$ $[0,1] \mapsto\left(x_{0}, \bar{x}(t)\right)$ to be a geodesic for the pseudo-metric 2.1 on $(N, h)$, so the path $\{\bar{x}(t) \mid 0 \leq t \leq 1\}$ appears convex from $x_{0}$.

We think of a function of the form $x \mapsto \lambda-c(x, \bar{y})$ as defining the elevation of a mountain on $M$, focused at (or indexed by) $\bar{y} \in \bar{M}$. The function $u(x)$ of (3.1) may be viewed as a double mountain, while the maximum 3.2 may be viewed as the upper envelope of a one-parameter family of mountains which slide as their foci $\bar{x}(t)$ move from $\bar{y}$ to $\bar{z}$. The proof of the preceding theorem relies on the fact that the sliding mountain stays beneath the double mountain (while remaining tangent to it at $x_{0}$ ), if the cost is weakly regular. In the applications below, we take $\Omega=\operatorname{cl} M$ and $\bar{\Omega}=\operatorname{cl} \bar{N}(x)=\operatorname{cl} \bar{M}$ tacitly.

Proof of Theorem 3.1. Let $c \in C^{4}(N)$ be weakly regular on some horizontally convex domain $N \subset M \times M$. Fix $u: \Omega \rightarrow \mathbb{R} \cup\{+\infty\}$ and $x \in \operatorname{Dom} u$ with $\bar{y}, \bar{z} \in \bar{\Omega} \cap \partial_{\Omega}^{c} u(x)$. This means $u(y) \geq u(x)-c(y, \bar{z})+c(x, \bar{z})$ for all $y \in \Omega$, the right hand side takes an unambiguous value in $\mathbb{R} \cup\{-\infty\}$, and the same inequality holds with $\bar{y}$ in place of $\bar{z}$. Apparent convexity of $\bar{\Omega}$ from $x$ implies there exists a geodesic $t \in] 0,1[\mapsto(x, \bar{x}(t))$ in $(N, h)$ with $\bar{x}(t) \in \bar{\Omega}$ which extends continuously to $\bar{x}(0)=\bar{y}$ and $\bar{x}(1)=\bar{z}$. The desired connectivity can be established by proving $\bar{x}(t) \in \partial_{\Omega}^{c} u(x)$ for each $\left.t \in\right] 0,1[$, since this means $\bar{\Omega} \cap \partial_{\Omega}^{c} u(x)$ appears convex from $x$.

Horizontal convexity implies $N(\bar{x}(t))$ appears convex from $\bar{x}(t)$ for each $t \in[0,1]$, so agrees with the illuminated set $V(x, \bar{x}(t))=N(\bar{x}(t))$ of Definition 4.7. For any $y \in$ $\bigcap_{0 \leq t \leq 1} N(\bar{x}(t))$, the sliding mountain lies below the double mountain, i.e., $f(t, y):=$ $-c(y, \bar{x}(t))+c(x, \bar{x}(t)) \leq \max \left\{f\left(0^{+}, y\right), f\left(1^{-}, y\right)\right\}$, according to Theorem 4.10 and Remark 4.12. Note that $f:[0,1] \times \Omega \rightarrow \mathbb{R} \cup\{-\infty\}$ is a continuous function, since 
$t \in[0,1] \mapsto(x, \bar{x}(t)) \in \operatorname{cl} N$ and $c: \operatorname{cl}(N) \rightarrow \mathbb{R} \cup\{+\infty\}$ are continuous and their composition is real-valued. We therefore replace $0^{+}$by 0 and $1^{-}$by 1 and extend the inequality to all $y \in \Omega$ using the density of $\bigcap_{0 \leq t \leq 1} N(\bar{x}(t))$. On the other hand, $\bar{x}(t) \in$ $\partial_{\Omega}^{c} u(x)$ if and only if $u(y) \geq u(x)+f(t, y)$ for each $y \in \Omega$ and $t \in[0,1]$. Since $u(y) \geq$ $u(x)+\max \{f(0, y), f(1, y)\}$ by hypothesis, we have established apparent convexity of $\bar{\Omega} \cap \partial_{\Omega}^{c} u(x)$ from $x$ and the proof is complete.

Remark 3.2 (Hölder continuity). Since connectedness and apparent convexity survive closure, we may replace $\bar{\Omega} \cap \partial_{\Omega}^{c} u(x)$ by its closure (often $\partial^{c} u(x)$ ) without spoiling the result. The apparent convexity of $\partial^{c} u(x)$ from $x$ hints at a kind of monotonicity for the correspondence $x \in M \mapsto \partial^{c} u(x)$. A strict form of this monotonicity can be established when the cost is strictly regular (A3s), and was exploited by Loeper to prove Hölder continuity $F \in C^{1 /(4 n-1)}(M ; \operatorname{cl} \bar{M})$ of the optimal map between densities $\rho \in L^{\infty}(M)$ and $\bar{\rho}$ with $1 / \bar{\rho} \in L^{\infty}(M)$ for costs which are strictly regular and bi-twisted on the closure of a bi-convex domain $M \times \bar{M} \subset \subset \mathbb{R}^{2 n}$. Details of his argument and conclusions can be found in [31], [32] or [27].

Remark 3.3 (On the relevance of twist and apparent convexity to the converse). In the absence of the twist condition, we have defined apparent convexity by the existence of a geodesic, which need not be extremal or unique. When (A3w) fails in this general setting, Loeper's converse argument shows the existence of a geodesic segment with endpoints in $(\{x\} \times \bar{N}(x)) \cap \partial^{c} u$ but which departs from this set at some points in between. Since the twist condition and apparent convexity imply the existence and uniqueness of geodesics linking points in $\{x\} \times \bar{N}(x)$, for a twisted cost the "if" statement in Theorem 3.1 becomes necessary as well as sufficient, a possibility which was partly anticipated in Ma, Trudinger \& Wang [34].

Remark 3.4 (Product domains). If $N=M \times \bar{M}$ the hypotheses and conclusions become simpler to state because $N(\bar{x})=M$ and $\bar{N}(x)=\bar{M}$ for each $(x, \bar{x}) \in N$. If, in addition the product $N=M \times \bar{M} \subset \mathbb{R}^{2 n}$ is a bounded Euclidean domain, we recover the result proved by Loeper [31] based on the regularity results of Trudinger \& Wang [42], whose hypotheses were relaxed after [43].

Example 3.5 (The reflector antenna and conformal geometry). The restriction of the cost function $c(x, \bar{x})=-\log |x-\bar{x}|$ from $\mathbb{R}^{n} \times \mathbb{R}^{n}$ to the unit sphere $M=\bar{M}=$ $\mathbb{S}^{n-1}:=\partial \mathbb{B}_{1}^{n}(0)$ arises in conjunction [53], [24] with the reflector antenna problem studied by Caffarelli, Glimm, Guan, Gutierrez, Huang, Kochengin, Marder, Newman, Oliker, Waltmann, Wang, and Wescott, among others. It induces a pseudo-metric $h$ known to satisfy (A0)-(A3s) with $N=(M \times \bar{M}) \backslash \Delta$. Note, however, that $c(x, \bar{x})$ actually defines a pseudo-metric $h^{(\infty, \infty)}$ on the larger space $\mathbb{R}^{n} \times \mathbb{R}^{n} \backslash \Delta$ which is almost but not quite bi-convex. Here $\Delta:=\left\{(y, y) \mid y \in \mathbb{R}^{n} \cup\{\infty\}\right\}$ denotes the diagonal. For fixed $a \neq \bar{a} \in \mathbb{R}^{n}$,

$$
\tilde{c}^{(a, \bar{a})}(x, \bar{x}):=-\frac{1}{2} \log \frac{|x-\bar{x}|^{2}|a-\bar{a}|^{2}}{|x-\bar{a}|^{2}|a-\bar{x}|^{2}}
$$


induces a pseudo-metric $h^{(a, \bar{a})}$ which coincides with $h^{(\infty, \infty)}$ on the set where both can be defined. Moreover, $\tilde{c}^{(a, \bar{a})}$ extends smoothly to $\left(\tilde{M}_{a} \times \tilde{M}_{\bar{a}}\right) \backslash \Delta$ where $\tilde{M}=\mathbb{R}^{n} \cup\{\infty\}$ is the Riemann sphere and $\tilde{M}_{a}=\tilde{M} \backslash\{\tilde{a}\}$. Furthermore $h^{(a, \bar{a})}$ is independent of $(a, \bar{a})$, so has a (unique) extension $\tilde{h}$ to $\tilde{N}:=(\tilde{M} \times \tilde{M}) \backslash \Delta$ which turns out to satisfy (A2)-(A3s) on the bi-convex set $\tilde{N}$; as in [31], [34], [42], this can be verified using the alternative characterization of (A3w/s) via concavity (/ 2-uniform concavity) of the restriction of the function

$$
q^{*} \in T_{x}^{*} \tilde{M} \mapsto p^{i} p^{j} c_{i j}\left(x, c-\operatorname{Exp}_{x} q^{*}\right)
$$

to the nullspace of $p \in T_{x} \tilde{M}$ in Dom $c$-Exp ; here the $c$-Exp map is defined at 4.3 . The nullspace condition is crucial, since the value of this function is given by

$$
\left.p^{i} p^{j} f_{i j}\right|_{(D f)^{-1}\left(-q^{*}\right)}=2\left(q_{i}^{*} p^{i}\right)^{2}-|p|^{2}\left|q^{*}\right|^{2}
$$

where the left hand expression in (3.4) coincides with the right hand expression in 3.3 for general costs of the form $c(x, \bar{x})=f(x-\bar{x})$. Homogeneity of the resulting manifold $(\tilde{N}, \tilde{h})$ follows from the symmetries $\tilde{N}=(F \times F)(\tilde{N})$ and

$$
\tilde{c}^{(a, \bar{a})}(x, \bar{x})=\tilde{c}^{(F(a), F(\bar{a}))}(F(x), F(\bar{x}))
$$

under simultaneous translation $F(x)=x-y$ by $y \in \mathbb{R}^{n}$ or inversion $F(x)=x /|x|^{2}$ of both factor manifolds; note the identity $|x-\bar{x}|=\left.|x||\bar{x}||| x\right|^{-2} x-|\bar{x}|^{-2} \bar{x} \mid$. This simplifies the verification of (A2)-(A3s), since it means infinity plays no distinguished role. Bi-convexity of $\tilde{N}$ follows from the fact that the projection of the null geodesic through $(z, \bar{x})$ and $(y, \bar{x})$ onto $M$ is given by a portion of the circle in $\mathbb{R}^{n} \cup\{\infty\}$ passing through $z, y$ and $\bar{x}$ - the unique arc of this circle (or line) stretching from $z$ to $y$ which does not pass through $\bar{x}$. From homogeneity it suffices to compute this geodesic in the case $(z, \bar{x})=(\infty, 0)$; we may further take $y=(1,0, \ldots, 0)$ using invariance under simultaneous rotations $F(x)=\Lambda x$ by $\Lambda \in O(n)$ and dilations $F(x)=\lambda x$ by $\lambda>0$. This calculation demonstrates that $\bigcap_{0 \leq t \leq 1} \tilde{N}(\bar{x}(t))$ is the complement of a circular archence a dense subset of $\tilde{M}$-for each geodesic $t \in[0,1] \mapsto(x, \bar{x}(t)) \in \tilde{N}$. Notice that if $x, \bar{x}(0), \bar{x}(1)$ all lie on the sphere $M=\bar{M}$, then so does $\bar{x}(t)$ for each $t \in[0,1]$. After verifying that $h$ coincides with the restriction of $\tilde{h}$ to the codimension-2 submanifold $N$, we infer for $\bar{x} \in \partial \mathbb{B}_{1}^{n}(0)$ that $N(\bar{x}) \times\{\bar{x}\}$ is a totally geodesic hypersurface in $\tilde{N}(\bar{x}) \times\{\bar{x}\}$, so the horizontal and vertical geodesics, bi-convexity, and strict regularity of $(N, h)$ are inherited directly from the geometry of $(\tilde{N}, \tilde{h})$, and the strict regularity via Lemma 4.5. Although we lack a globally defined smooth cost on $\tilde{N}$, we have one on $N$, so the hypotheses and hence the conclusions of Theorems 3.1 and 4.10 are directly established in the reflector antenna problem, whose geometry is also clarified: the vertical geodesics are products of points on $M$ with circles on $\bar{M}$, where by circle we mean the intersection of a two-dimensional plane with $\bar{M}=\partial \mathbb{B}_{1}^{n}(0)$. The geodesics would be the same for the negation $c_{+}(x, y)=+\log |x-y|$ of this cost, which satisfies (A0)(A2) on the bi-convex domain $N$, but violates (A3w) for the same reason that $c$ satisfies (A3s). Using the Euclidean norm on our coordinates, the $c$-exponential 4.3 is given by $\bar{x}=c-\operatorname{Exp}_{x}\left(q^{*}\right):=x-q^{*} /\left|q^{*}\right|^{2}$, the optimal map takes the form $F(x)=c$-Exp $\operatorname{Ex}_{x} D u(x)$, 
and the resulting degenerate elliptic Monge-Ampère type equation (5.1)-(5.2) on $\mathbb{R}^{n}$, expressed in coordinates, is

$$
\operatorname{det}\left[u_{i j}(x)+2 u_{i}(x) u_{j}(x)-\delta_{i j}|D u(x)|^{2}\right]=\frac{|D u(x)|^{2 n} \rho(x)}{\bar{\rho}\left(x-D u(x) /|D u(x)|^{2}\right)} .
$$

Here $\bar{\rho}(\bar{x})=O\left(|\bar{x}|^{-2 n}\right)$ as $\bar{x} \rightarrow \infty$ since $\bar{\rho}$ transforms like an $n$-form under coordinate changes. Using the isometries above to make $D u\left(x_{0}\right)=0$ at a point $x_{0}$ of interest, a slight perturbation of the standard Monge-Ampère equation is recovered nearby. The operator under the determinant $\sqrt{3.5}$ is proportional to the Schouten tensor of a conformally flat metric $d s^{2}=e^{-4 u} \sum_{i=1}^{n}\left(d x^{i}\right)^{2}$, so a similar equation occurs in Viaclovsky's $\sigma_{n}$-version of the Yamabe problem [45], [46], which has been studied by the many authors in conformal geometry surveyed in [47] and [40].

Example 3.6 (Riemannian manifolds). Consider a Riemannian manifold $(M=\bar{M}, g)$. Taking the cost function to be the square of the geodesic distance $c(x, \bar{x})=d^{2}(x, \bar{x}) / 2$ associated to $g$, induces a pseudo-metric tensor (2.1) on the domain $N$ where $c(x, \bar{x})$ is smooth, i.e. the complement of the cut locus. Moreover, the cost exponential (Definition [4.3) reduces [36] to the Riemannian exponential

$$
c-\operatorname{Exp}_{x} p^{*}=\exp _{x} p
$$

with the metrical identification $p^{*}=g(p, \cdot)$ of tangent and cotangent space. A curve $t \in[0,1] \mapsto x(t) \in N(\bar{x})$ through $\bar{x}$ is a geodesic in $(M, g)$ if and only if the curve $\tau(t)=(x(t), \bar{x})$ is a (null) geodesic in $(N, h)$, according to Lemma 4.4. On the diagonal $x=\bar{x}$, we compute $h(p \oplus \bar{p}, p \oplus \bar{p})=g(p, \bar{p})$, meaning the pseudo-Riemannian space $(N, h)$ contains an isometric copy of the Riemannian space $(M, g)$ along its diagonal $\Delta:=\{(x, x) \mid x \in M\}$. The symmetry $c(x, \bar{x})=c(\bar{x}, x)$ shows $\Delta$ to be embedded in $N$ as a totally geodesic submanifold, and nullity of $p \oplus \bar{p} \in T_{(x, x)} N$ reduces to orthogonality of $p$ with $\bar{p}$. This perspective illuminates Loeper's observation [31] that negativity of one Riemannian sectional curvature at any point on $(M, g)$ violates weak regularity $(\mathbf{A 3} \mathbf{w})$ of the cost. Indeed, the comparison of 4.9 ) with Lemma 4.5 allows us to recover the fact that along the diagonal, cross-curvatures in $(N, h)$ are proportional to Riemannian curvatures in $(M, g)$ :

$$
\sec _{(x, x)}^{(N, h)}(p \oplus 0) \wedge(0 \oplus \bar{p})=\frac{4}{3} \sec _{x}^{(M, g)} p \wedge \bar{p} .
$$

Example 3.7 (The round sphere). In the case of the sphere $M=\bar{M}=\mathbb{S}^{n}$ equipped with the standard round metric, the cut locus consists of pairs $(x, \bar{x})$ of antipodal points $d(x, \bar{x})=$ Diam $M$. Denote its complement by $N=\{(x, \bar{x}) \mid d(x, \bar{x})<$ Diam $M\}$, where $c(x, \bar{x})=d^{2}(x, \bar{x}) / 2$ is smooth. In this case, the identification 3.6 of cost exponential with Riemannian exponential implies bi-convexity of $N$, since the cut locus forms a circle (hypersphere if $n>2$ ) in the tangent space $T_{x} \mathbb{S}^{n}$, and the verification of (A3s) both on and off the diagonal was carried out by Loeper [32]. In fact more is true: $(N, h)$ is non-negatively cross-curved, as we verify in [29]. Given an $h$-geodesic $t \in[0,1] \mapsto$ $(x, \bar{x}(t)) \in N$, we find $\bigcap_{0 \leq t \leq 1} N(\bar{x}(t))$ exhausts $\mathbb{S}^{n}$ except for the antipodal curve to the 
exponential image $t \in[0,1] \mapsto \bar{x}(t)$ of a line segment in $T_{x} \mathbb{S}^{n}$. Although this curve does not generally lie on a great circle or even a circle, its complement is dense in $\mathbb{S}^{n}$, whence the double-mountain above sliding-mountain property and Theorem 3.1 follow.

Example 3.8 (Positive versus negative curvature). To provide more insight into Loeper's results - continuity of optimal maps on the round sphere versus discontinuous optimal maps on the saddle or hyperbolic plane - consider dividing a smooth positive density $\rho$ (say uniform on some disk of volume $\sum_{i=1}^{3} \epsilon_{i}=1$ ) optimally between three points $\bar{x}_{1}, \bar{x}_{2}, \bar{x}_{3}$ on a geodesic through its centre: $\bar{\rho}(\cdot)=\sum_{i=1}^{3} \epsilon_{i} \delta_{\bar{x}_{i}}(\cdot)$. The solution to this problem is given, e.g. [22], by finding constants $\lambda_{1}, \lambda_{2}, \lambda_{3} \in \mathbb{R}$ for which the function

$$
u(x)=\max _{1 \leq i \leq 3}\left\{u_{i}(x)\right\} \quad \text { given by } \quad u_{i}(x):=-\lambda_{i}-c\left(x, \bar{x}_{i}\right)
$$

solves

$$
\epsilon_{i}=\rho\left[\Omega_{i}\right] \quad \text { with } \quad \Omega_{i}:=\left\{x \in M \mid u(x)=u_{i}(x)\right\}
$$

for $i=1,2,3$. The regions $\Omega_{i}$ are illustrated in Figure 1 for the case where the cost $c(x, \bar{x})=d^{2}(x, \bar{x}) / 2$ is proportional to Riemannian distance squared on the (a) round sphere, (b) Euclidean plane, (c) hyperbolic disc.

(a)

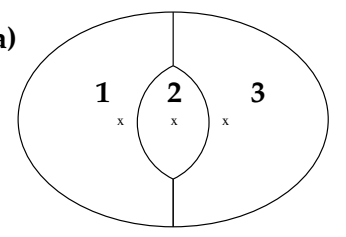

(b)

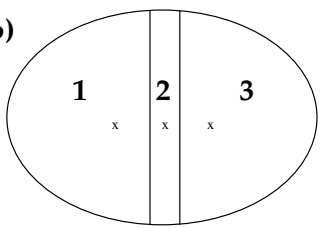

(c)

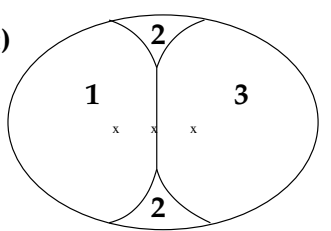

Fig. 1

Notice that only in case (c) is one of the regions $\Omega_{2}$ disconnected; in cases (a) and (b) all regions $\Omega_{i}$ must be connected by the preceding result and example. Now consider transporting a smoothly smeared and positive approximation $\bar{\rho}_{\eta}(\cdot)$ of the discrete source $\bar{\rho}(\cdot)$, obtained by mollifying on scale $\eta>0$, to the uniform measure $\rho(\cdot)$. For $\eta>0$ sufficiently small, points $\bar{x}$ near $\bar{x}_{2}$ will need to map into a $\delta$-neighbourhood of $\Omega_{2}$. In case (c), recalling Loeper's argument we can now assert that if $\eta>0$ is small the optimal map $G_{\eta}$ from $\bar{\rho}_{\eta}(\cdot)$ to $\rho(\cdot)$ will have a discontinuity near $\bar{x}_{2}$ separating the regions which map into disjoint $\delta$-neighbourhoods separating the two disconnected components of $\Omega_{2}$. If this were not the case, mass balance implies for small $\eta$ this map $G_{\eta}=\check{c}$-Exp $\circ D \bar{u}_{\eta}$ would need to cross the horizontal midline in Figure 1(c) at some $x_{\eta}=G_{\eta}\left(\bar{x}_{\eta}\right)$ with $\bar{x}_{\eta}$ near $\bar{x}_{2}$. Here $\breve{c}(\bar{x}, x):=c(x, \bar{x})$. Since the potentials $\bar{u}_{\eta}$ have a semiconvexity constant independent of $\eta>0$, taking a limit implies $\partial^{\check{c}} \bar{u}\left(x_{2}\right)$ intersects the midline of the closure of our unit volume hyperbolic disk at some subsequential limit point $x_{0}=\lim _{\eta_{k} \rightarrow 0} x_{\eta_{k}}$. This contradicts a direct calculation starting from (3.8), which shows the horizontal axis remains disjoint from region $\Omega_{2}$ in case (c). 
Example 3.9 (New examples from old: perturbations, submersions, and products). It remains interesting to find more general sufficient conditions on a Riemannian manifold $(M, g)$ and function $f$ for the pseudo-metric $h$ induced on the complement of the cut locus $N \subset M \times M$ by $c(x, \bar{x})=f(d(x, \bar{x}))$ to be strictly or weakly regular. It is clear that slight perturbations of an (A3s) cost remain strictly regular locally if the perturbation is smooth and small enough. Delanoë \& Ge have quantified this observation away from the cut locus on the round sphere [15], while the subtleties associated with the cut locus have been resolved on $\mathbb{S}^{2}$ by Figalli \& Rifford [19]; see also Loeper \& Villani [33]. It is also possible to deduce that the local properties such as (A3s/w) and non-negative cross-curvature and the global property such as the conclusion of Theorem 3.1 all survive Riemannian submersion [29], holding for example on quotients of the round sphere under discrete [15], [29] or continuous [29] group actions, including in particular all spaces of constant positive curvature [15], and the Fubini-Study metric on $\mathbb{C P}^{n}$ [29]. On the other hand, an example constructed by one of us shows that positive but non-constant sectional curvature of the underlying manifold $(M=\bar{M}, g)$ does not guarantee weak regularity of the cost $c=d^{2} / 2$ away from the diagonal in $N \subset M \times M$ [26]. As a final important example, consider two manifolds $N_{+} \subset M_{+} \times \bar{M}_{+}$and $N_{-} \subset M_{-} \times \bar{M}_{-}$equipped with cost functions $c_{ \pm} \in C^{4}\left(N_{ \pm}\right)$inducing pseudo-metrics $d \ell_{ \pm}^{2}=-c_{i \bar{j}} d x_{ \pm}^{i} d x_{ \pm}^{\bar{j}}$. As a consequence of Lemma 4.5 the product metric $d \ell^{2}=d \ell_{+}^{2}+d \ell_{-}^{2}$ corresponding to the cost function $c_{+}\left(x_{+}, \bar{x}_{+}\right)+c_{-}\left(x_{-}, \bar{x}_{-}\right)$on $N=N_{+} \times N_{-}$is non-negatively crosscurved 2.3 if both $\left(N_{ \pm}, h_{ \pm}\right)$are. Although it is not true that weak regularity of the factors implies the same for the product, many of the known examples of weakly regular costs (including those of Examples 2.4, 3.7 and the sumbersions above) actually turn out to be non-negatively cross-curved [29], so this product construction becomes a fruitful source of new examples. Furthermore, since geodesics in the product are products of geodesics, bi-convexity of the factors $\left(N_{ \pm}, h_{ \pm}\right)$implies bi-convexity of the product manifold $(N, h)$. Because a product geodesic may have constant factors, it is not hard to show that the non-negatively curved product manifolds $(N, h)$ always fail to be strictly regular even when both factor manifolds $\left(N_{ \pm}, h_{ \pm}\right)$are positively cross-curved. Thus tensor products of positively cross-curved costs yield a new source of weakly regular costs that fail to be strictly regular - the very simplest example of which is given by arbitrary sums $c(x, \bar{x})=\sum_{k=1}^{n} c\left(x^{k}, x^{\bar{k}} ; k\right)$ of $k=1, \ldots, n$ positively cross-curved costs $c(s, t ; k)$ as in 2.7) on bi-convex subdomains $N_{1}, \ldots, N_{n} \subset \mathbb{R}^{2}$.

Remark 3.10 (Products $\mathbb{S}^{n_{1}} \times \cdots \times \mathbb{S}^{n_{k}} \times \mathbb{R}^{l}$ and their Riemannian submersions). The conclusion of Theorem 3.1 holds for the distance squared cost on the Riemannian product $M=\bar{M}$ of round spheres $\mathbb{S}^{n_{1}} \times \cdots \times \mathbb{S}^{n_{k}} \times \mathbb{R}^{l}$ - or its Riemannian submersions-by combining the preceding example with the result of [29]. The weak regularity (A3w) of the cost on $N=M \times \bar{M} \backslash\{$ cut locus\} and the biconvexity of $N$ are satisfied as in Example 3.9. The density condition of $\bigcap_{0 \leq t \leq 1} N(\bar{x}(t))$ also follows easily since the cut locus of one point in this example is a smooth submanifold of codimension greater than or equal to 2. This new global result illustrates an advantage of our method over other approaches [42], [43], [31], [32], which would require a regularity result for optimal maps (or some a priori estimates) to obtain the conclusion of Theorem 3.1. To implement such 
approaches for the manifolds of this example, one would need to establish that an optimal map remains uniformly away from the cut locus, as is currently known only for a single sphere $M=\bar{M}=\mathbb{S}^{n}$ from work of Delanöe \& Loeper [16] (and can also be obtained [27] by an approach based on [31] instead). To the best of our knowledge, no one has yet succeeded in establishing regularity results for this product example, though one could try to obtain them from Theorem 3.1, by extending the approach we develop successfully in [27] for a single sphere $M=M=\mathbb{S}^{n}$.

\section{Proof of main results}

Let us begin by establishing coordinate representations of the Christoffel symbols and Riemann curvature tensor for the pseudo-metric 2.1 . Standard references for pseudoRiemannian geometry include the books of Wald [51] and O'Neill [39], though it is called semi-Riemannian geometry there.

Lemma 4.1 (Riemann curvature tensor and Christoffel symbols). Use a non-degenerate cost $c \in C^{4}(N)$ to define a pseudo-metric 2.1) on the domain $N \subset M \times \bar{M}$. In local coordinates $x^{1}, \ldots, x^{n}$ on $M$ and $x^{\overline{1}}, \ldots, x^{\bar{n}}$ on $\bar{M}$, the only non-vanishing Christoffel symbols are

$$
\Gamma_{i j}^{m}=c^{m \bar{k}} c_{\bar{k} i j} \quad \text { and } \quad \Gamma_{\bar{i} \bar{j}}^{\bar{m}}=c^{\bar{m} k} c_{k \bar{i} \bar{j}} .
$$

Furthermore, the components of the Riemann curvature tensor 2.2 vanish except when the number of barred and unbarred indices is equal, in which case the value of the component can be inferred from $R_{i j \bar{k} \bar{l}}=0$ and

$$
2 R_{i \bar{j} \bar{k} l}=c_{i \bar{j} \bar{k} l}-c_{l i \bar{f}} c^{a \bar{f}} c_{a \bar{j} \bar{k}} .
$$

Proof. Following (3.1.3) and (3.4.4) of e.g. [51],

$$
\Gamma_{i j}^{m}:=\frac{1}{2} h^{m k}\left(h_{k j, i}+h_{i k, j}-h_{i j, k}\right)+\frac{1}{2} h^{m \bar{k}}\left(h_{\bar{k} j, i}+h_{i \bar{k}, j}-h_{i j, \bar{k}}\right),
$$

and the analogous definition with $i, j$, and/or $m$ replaced by $\bar{i}, \bar{j}$ and $\bar{m}$ respectively, the off-diagonal form 2.1 of the pseudo-metric and the equality of mixed partials implies the only non-vanishing Christoffel symbols are given by

$$
\Gamma_{i j}^{m}=-\frac{1}{2} c^{m \bar{k}}\left(-c_{\bar{k}_{j i}}-c_{i \bar{k} j}+0\right)=c^{m \bar{k}} c_{\bar{k} i j}
$$

and $\Gamma_{\bar{i} \bar{j}}^{\bar{k}}=c^{\bar{m} k} c_{k \bar{i}} \bar{j}$. Since the only non-vanishing Christoffel symbols are given by 4.1 , it is not hard to compute the relevant components of Riemann's curvature tensor in the coordinates we have chosen:

$$
\begin{aligned}
R_{i \bar{j} \bar{k}}^{\bar{m}} & =-\frac{\partial}{\partial x^{i}} \Gamma_{\bar{j} \bar{k}}^{\bar{m}}+\frac{\partial}{\partial x_{\bar{j}}} \Gamma_{i \bar{k}}^{\bar{m}}+\Gamma_{\bar{k} i}^{f} \Gamma_{\bar{j} f}^{\bar{m}}-\Gamma_{\bar{k} \bar{j}}^{f} \Gamma_{i f}^{\bar{m}}+\Gamma_{\bar{k} i}^{\bar{f}} \Gamma_{\bar{j} \bar{f}}^{\bar{m}}-\Gamma_{\bar{k} \bar{j}}^{\bar{f}} \Gamma_{i \bar{f}}^{\bar{m}} \\
& =-\frac{\partial}{\partial x^{i}}\left(c^{\bar{m} a} c_{a \bar{j} \bar{k}}\right)=-c^{\bar{m} b} c_{i \bar{j} \bar{k} b}+c^{\bar{m} b} c_{b i \bar{f}} c^{a \bar{f}} c_{a \bar{j} \bar{k}} .
\end{aligned}
$$


Using the pseudo-metric 2.1 to lower indices yields 4.2, and the other non-vanishing components of the Riemann tensor can then be deduced from the well-known symmetries $-R_{\bar{j} i \bar{k} l}=R_{i \bar{j} \bar{k} l}=R_{\bar{k} l i \bar{j}}=-R_{l \bar{k} i \bar{j}}$. To see that the remaining components all vanish, it suffices to repeat the analysis starting from the analogous definitions of $R_{i j \bar{k}}{ }^{\bar{m}}, R_{i j \bar{k}}{ }^{m}$, and $R_{i j k}{ }^{\bar{m}}$.

Remark 4.2 (Vanishing curvatures). The vanishing of $R_{i j k l}, R_{i j k \bar{l}}, R_{i \bar{j} \bar{k} \bar{l}}, R_{\bar{i} \bar{j} \bar{k} \bar{l}}$, and $R_{i j \bar{k} \bar{l}}=0$ imply that $\left(\bigwedge^{2} T M\right) \oplus\left(\bigwedge^{2} T \bar{M}\right)$ lies in the null space of curvature operator viewed as a quadratic form on $\bigwedge^{2} T N=\left(\bigwedge^{2} T M\right) \oplus\left(\bigwedge^{2} T \bar{M}\right) \oplus(T M \wedge T \bar{M})$. Strict/weak regularity of the cost, and the signs of the cross-curvature $(2.3)$, and sectional curvature 2.4 are therefore all determined by the action of this operator on the $n^{2}$-dimensional vector bundle $T M \wedge T \bar{M}$. Since the cone of null vectors is non-linear, it is not obvious whether (A3w) implies non-negativity of all cross-curvatures of $(N, h)$, but Trudinger \& Wang [42] prove, as in Example 3.5, that this is not so.

We next recall an important map of Ma, Trudinger \& Wang [34], called the costexponential by Loeper [31].

Definition 4.3 (Cost exponential). If $c \in C^{2}(N)$ is twisted (A1), we define the $c$-exponential on

$$
\begin{aligned}
\operatorname{Dom}\left(c-\operatorname{Exp}_{x}\right): & =-D c(x, \bar{N}(x)) \\
& =\left\{p^{*} \in T_{x}^{*} M \mid p^{*}=-D c(x, \bar{x}) \text { for some } \bar{x} \in \bar{N}(x)\right\}
\end{aligned}
$$

by $c$-Exp $p_{x}^{*}=\bar{x}$ if $p^{*}=-D c(x, \bar{x})$. Non-degeneracy (A2) then implies the $c$-exponential is a diffeomorphism from $\operatorname{Dom}\left(c-\operatorname{Exp}_{x}\right) \subset T_{x}^{*} M$ onto $\bar{N}(x) \subset \bar{M}$. If $c \in C^{2}(N)$ is non-degenerate but not twisted and $q^{*}=-D c(x, \bar{y})$, the implicit function theorem implies a single-valued branch of $c$-Exp $x$ taking values near $\bar{y}$ is defined by the same formula in a small neighbourhood of $q^{*}$, though it no longer extends to be a global diffeomorphism of $\operatorname{Dom}\left(c-\operatorname{Exp}_{x}\right)$ onto $\bar{N}(x)$.

Lemma 4.4 (The $c$-segments of [34] are geodesics). Use a non-degenerate cost $c \in$ $C^{4}(N)$ to define a pseudo-metric 2.1] on the domain $N \subset M \times \bar{M}$. Fix $x \in M$. If $p^{*}, q^{*} \in$ $\operatorname{Dom}\left(c-\operatorname{Exp}_{x}\right) \subset T_{x}^{*} M$ are close enough, there is a branch of $c-\operatorname{Exp}_{x}$ defined on the line segment joining $p^{*}$ to $q^{*}$. Then the curve $s \in[0,1] \mapsto \sigma(s):=\left(x, c-\operatorname{Exp}_{x}\left((1-s) p^{*}+\right.\right.$ $\left.s q^{*}\right)$ ) is an affinely parameterized null geodesic in $(N, h)$. Conversely, every geodesic segment in the totally geodesic submanifold $\{x\} \times \bar{N}(x)$ can be parameterized locally in this way.

Proof. Given $s_{0} \in[0,1]$, introduce coordinates on $M$ and $\bar{M}$ around $\sigma\left(s_{0}\right)$ so that nearby, the curve $\sigma(s)$ can be represented in the form $\left(x^{1}, \ldots, x^{n}, x^{\overline{1}}(s), \ldots, x^{\bar{n}}(s)\right)$. Differentiating the definition of the cost exponential

$$
0=(1-s) p_{i}^{*}+s q_{i}^{*}+c_{i}(\sigma(s))
$$

twice with respect to $s$ yields

$$
0=c_{i j} \ddot{j}^{\bar{k}}+c_{i \bar{j} \bar{k}} \dot{x}^{\bar{j}} \dot{x}^{\bar{k}}
$$


for each $i=1, \ldots, n$. Multiplying by the inverse matrix $c^{\bar{m} i}$ to $c_{i} \bar{j}$ yields

$$
0=\ddot{x}^{\bar{m}}+c^{\bar{m} i} c_{i \bar{j} \bar{k}} \dot{x}^{\bar{j}} \dot{x}^{\bar{k}},
$$

always summing on repeated indices. Together with $\ddot{x}^{m}=0=\dot{x}^{m}$, we claim these reduce to the geodesic equations (e.g. (3.3.5) of [51]),

$$
\begin{aligned}
& 0=\ddot{x}^{m}+\Gamma_{i j}{ }^{m} \dot{x}^{i} \dot{x}^{j}+\Gamma_{\bar{i} j}{ }^{m} \dot{x}^{\bar{i}} \dot{x}^{j}+\Gamma_{i}{ }^{m} \dot{x}^{i} \dot{x}^{\bar{j}}+\Gamma_{\bar{i} \bar{j}}{ }^{m} \dot{x}^{\bar{i}} \dot{x}^{\bar{j}}, \\
& 0=\ddot{x}^{\bar{m}}+\Gamma_{i j}{ }^{\bar{m}} \dot{x}^{i} \dot{x}^{j}+\Gamma_{\bar{i} j}{ }^{\bar{m}} \dot{x}^{\bar{i}} \dot{x}^{j}+\Gamma_{i} \bar{j}^{\bar{m}} \dot{x}^{i} \dot{x}^{\bar{j}}+\Gamma_{\bar{i}} \bar{j}^{\bar{j}} \dot{x}^{\bar{i}} \dot{x}^{\bar{j}},
\end{aligned}
$$

on $(N, h)$. Indeed, this follows since the only non-vanishing Christoffel symbols are given by (4.1). Comparing (4.6) with (4.7), we see $\sigma(s)$ is an affinely parameterized geodesic near $\sigma\left(s_{0}\right)$; it is null since $h(\dot{\sigma}, \dot{\sigma})=0$ from the off-diagonal form of 2.1.

Conversely, any geodesic segment in $(N, h)$ which lies in $x \times \bar{N}(x)$ can be parameterized affinely on $s \in[0,1]$. Near $s_{0} \in[0,1]$ it then satisfies 44.5$]$, whence

$$
0=\frac{d^{2}}{d s^{2}} c_{i}(x, \bar{x}(s)) .
$$

Integrating twice, the constants of integration determine $p^{*}, q^{*} \in T_{x}^{*} M$ such that 4.4 holds locally. Thus $\left(1-s_{0}\right) p^{*}+s_{0} q^{*} \in \operatorname{Dom}(c$-Exp $x)$. Choosing a branch of the cost exponential defined near this point and equalling $D_{i} c\left(x, \bar{x}\left(s_{0}\right)\right)$ there, we deduce $(x, \bar{x}(s))$ $=c-\operatorname{Exp}_{x}\left((1-s) p^{*}+s q^{*}\right)$ for $s$ near $s_{0}$ from the definition of this branch.

Finally, to see that $\{x\} \times \bar{N}(x)$ is totally geodesic, take any point $\bar{x} \in \bar{N}(x)$ and tangent vector $\bar{p} \in T_{\bar{x}} \bar{M}$. Setting $x^{m}(s)=x^{m}$ to be constant solves half of the geodesic equations, since $\Gamma_{\bar{i} j}^{m}=0=\Gamma_{\bar{i} \bar{j}}^{m}$. we can still solve the remaining $n$ components of the geodesic equation (4.6) for small $s \in \mathbb{R}$, subject to the initial conditions $\bar{x}(0)=\bar{x}$ and $\dot{\bar{x}}(0)=q$, to find a geodesic which remains in the $n$-dimensional submanifold $\{x\} \times \bar{N}(x)$ for short times.

The next lemma gives a non-tensorial expression of the sectional curvature in our pseudoRiemannian geometry $(N, h)$. In the context of Example 3.6, it can be viewed as a generalization of the asymptotic formula for the Riemannian distance between two arclength parameterized geodesics $x(s)$ and $\bar{x}(t)$ near a point of intersection $x(0)=\bar{x}(0)$ at angle $\theta$ :

$$
d^{2}(x(s), \bar{x}(t))=s^{2}+t^{2}-2 s t \cos \theta-\frac{k}{3} s^{2} t^{2} \sin ^{2} \theta+O\left(\left(s^{2}+t^{2}\right)^{5 / 2}\right)
$$

where the Riemannian curvature $k$ of the two-plane $\dot{x}(0) \wedge \dot{\bar{x}}(0)$ on $(M=\bar{M}, g)$ gives the leading order correction to the law of cosines. Though we do not need it here, the proof of the next lemma can also be adapted to establish an expansion analogous to (4.9) for general costs $c(x(s), \bar{x}(t))$; the zeroth and first order terms do not vanish, but the coefficients of $s^{2} t$ and $s^{3} t$ are zero due to the geodesy of $s \in[0,1] \mapsto \sigma(s)$. Remarkably however, to determine the coefficient of $s^{2} t^{2}$ in the lemma below requires only one (in fact, either one) and not both of the two curves to be geodesic. 
Lemma 4.5 (Non-tensorial expression for curvature). Use a non-degenerate cost $c \in$ $C^{4}(N)$ to define a pseudo-metric 2 2.1] on the domain $N \subset M \times \bar{M}$. Let $(s, t) \in[-1,1]^{2}$ $\mapsto(x(s), \bar{x}(t)) \in N$ be a surface containing two curves $\sigma(s)=(x(s), \bar{x}(0))$ and $\tau(t)=$ $(x(0), \bar{x}(t))$ through $(x(0), \bar{x}(0))$. Note $0 \oplus \dot{\bar{x}}(0)$ defines a parallel vector field along $\sigma(s)$. If $s \in[-1,1] \mapsto \sigma(s) \in N$ is a geodesic in $(N, h)$ then

$$
-\left.2 \frac{\partial^{4}}{\partial s^{2} \partial t^{2}}\right|_{s=0=t} c(x(s), \bar{x}(t))=\sec _{(x(0), \bar{x}(0))} \frac{d \sigma}{d s} \wedge \frac{d \tau}{d t} .
$$

Proof. Introduce coordinates $x^{1}, \ldots, x^{n}$ in a neighbourhood of $x(0)$ on $M$ and $x^{\overline{1}}, \ldots, x^{\bar{n}}$ in a neighbourhood of $\bar{x}(0)$ on $\bar{M}$, so the surface $(x(s), \bar{x}(t)) \in N$ has coordinates $\left(x^{1}(s), \ldots, x^{n}(s), x^{\overline{1}}(t), \ldots, x^{\bar{n}}(t)\right)$ locally. To see $0 \oplus \dot{\bar{x}}(0)$ defines a parallel vector field along $\sigma(s)$, we use the Levi-Civita connection to compute

$$
\dot{\sigma}^{i} \nabla_{i} \dot{x}^{\bar{k}}+\dot{\sigma}^{\bar{i}} \nabla_{\bar{i}} \dot{x}^{\bar{k}}=\dot{x}^{i} \frac{\partial \dot{x}^{\bar{k}}}{\partial x^{i}}+\dot{x}^{i} \Gamma_{i j}^{\bar{k}} \dot{x}^{j}+\dot{x}^{i} \Gamma_{i \bar{j}}^{\bar{k}} \dot{x}^{\bar{j}}+0=0
$$

since the only non-vanishing Christoffel symbols are given by 4.1].

Computing the fourth mixed derivative yields

$$
\begin{aligned}
\left.\frac{\partial^{4}}{\partial s^{2} \partial t^{2}}\right|_{s=0=t} c(x(s), \bar{x}(t)) & =c_{i j \bar{k} \bar{l}} \dot{x}^{i} \dot{x}^{j} \dot{x}^{\bar{k}} \dot{x}^{\bar{l}}+c_{a \bar{k} l} \ddot{x}^{a} \dot{x}^{\bar{k}} \dot{x}^{\bar{l}}+\left(c_{i j \bar{b}} \dot{x}^{i} \dot{x}^{j}+c_{a \bar{b}} \ddot{x}^{a}\right) \ddot{x}^{\bar{b}} \\
& =\left(c_{i \bar{k} \bar{l} j}-c_{\bar{k} \bar{l} a} c^{a \bar{b}} c_{\bar{b} i j}\right) \dot{x}^{i} \dot{x}^{j} \dot{x}^{\bar{k}} \dot{x}^{\bar{l}}
\end{aligned}
$$

where the form (4.6) of the geodesic equation has been used to eliminate the coefficient of $\ddot{x}^{\bar{b}}$ and express $\ddot{x}^{a}$ in terms of $\dot{x}^{i}$. Comparing 4.11, with 4.2, and 2.2 yields the desired conclusion 4.10 . The minus sign comes from antisymmetry $R_{i \bar{k} \bar{j} j}=-R_{i \bar{k} j \bar{l}}$ of the Riemann tensor.

Our next contribution culminates in Theorem 4.10 , which generalizes the result that Loeper [31], [32] deduced from Trudinger \& Wang [42]. As mentioned above, it can be interpreted to mean that if a weakly regular function $c \in C^{4}(N)$ governs the cost of transporting a commodity from the locations where it is produced to the locations where it is consumed, a shipper indifferent between transporting the commodity from $y$ to the consumer at either endpoint $\bar{x}(0)$ and $\bar{x}(1)$ of the geodesic $t \in[0,1] \mapsto(y, \bar{x}(t))$ in $(N, h)$, will also be indifferent to transporting goods from $y$ to the consumers at each of the intermediate points $\bar{x}(t)$ along this geodesic. As was also mentioned, for non-degenerate costs Loeper showed this conclusion fails unless the cost is weakly regular.

Proposition 4.6 (Maximum principle). Use a weakly regular cost $c \in C^{4}(N)$ to define a pseudo-metric 2.1 on the domain $N \subset M \times \bar{M}$. Given $x \neq y \in M$, let $t \in] 0,1[\mapsto$ $(x, \bar{x}(t)) \in N$ be a geodesic in $(N, h)$ with $\dot{\bar{x}}(1 / 2) \neq 0$ and set $f(t)=-c(y, \bar{x}(t))+$ $c(x, \bar{x}(t))$. If $\dot{f}\left(t_{0}\right)=0$ for some $\left.t_{0} \in\right] 0,1\left[\right.$ with a geodesic linking $\left(x, \bar{x}\left(t_{0}\right)\right)$ to $\left(y, \bar{x}\left(t_{0}\right)\right)$ lying in $N\left(\bar{x}\left(t_{0}\right)\right) \times\left\{\bar{x}\left(t_{0}\right)\right\}$, then $\ddot{f}\left(t_{0}\right) \geq 0$. Strict inequality holds if the relevant crosscurvature of $c$ is positive at some point on the second geodesic. 
Proof. Suppose $f(t)$ has a critical point at some $0<t_{0}<1$, so

$$
0=\dot{f}\left(t_{0}\right)=\left(\partial_{\bar{i}} c\left(x, \bar{x}\left(t_{0}\right)\right)-\partial_{\bar{i}} c\left(y, \bar{x}\left(t_{0}\right)\right)\right) \dot{x}^{\bar{i}}\left(t_{0}\right) ;
$$

we then claim $\ddot{f}\left(t_{0}\right) \geq 0$.

Let $s \in[0,1] \mapsto\left(x(s), \bar{x}\left(t_{0}\right)\right)$ be a geodesic in $N\left(\bar{x}\left(t_{0}\right)\right) \times\left\{\bar{x}\left(t_{0}\right)\right\}$ with endpoints $x(0)=x$ and $x(1)=y$. Set $g(s, t)=-c(x(s), \bar{x}(t))+c(x, \bar{x}(t))$. Lemma 4.5 yields

$$
\left.\frac{\partial^{4} g}{\partial s^{2} \partial t^{2}}\right|_{\left(s, t_{0}\right)}=\frac{1}{2} \sec _{\left(x(s), \bar{x}\left(t_{0}\right)\right)}(\dot{x}(s) \oplus 0) \wedge\left(0 \oplus \dot{\bar{x}}\left(t_{0}\right)\right) \geq 0,
$$

with the inequality following from weak regularity (A3w) of the cost $c \in C^{4}(N)$, as long as $\dot{x}(s) \oplus 0_{\bar{x}\left(t_{0}\right)}$ and $0_{x(s)} \oplus \dot{\bar{x}}\left(t_{0}\right)$ are orthogonal vectors on $(N, h)$, or equivalently, as long as $\dot{x}(s) \oplus \dot{\bar{x}}\left(t_{0}\right)$ is null. These vectors are non-vanishing since $x(0) \neq x(1)$ and $\dot{\bar{x}}(0) \neq 0$; we now deduce their orthogonality from $\sqrt{4.12}$, using subscripts to distinguish which tangent space the zero vectors reside in.

Along the geodesic $s \in[0,1] \mapsto\left(x(s), \bar{x}\left(t_{0}\right)\right)$, the vector field $0_{x(s)} \oplus \dot{\bar{x}}\left(t_{0}\right)$ is parallel transported according to Lemma 4.5 Thus the inner product $\lambda$ of this vector field with the tangent vector is independent of $s \in[0,1]$. Define $q_{\bar{i}}^{*}(s):=\partial_{\bar{i}} c\left(x(s), \bar{x}\left(t_{0}\right)\right) \in T_{\bar{x}\left(t_{0}\right)}^{*} \bar{M}$, so $\dot{q}_{\dot{i}}^{*}(s)=\partial_{j} \partial_{\bar{i}} c\left(x(s), \bar{x}\left(t_{0}\right)\right) \dot{x}^{j}(s)$. From the form 2.1 of the pseudo-metric we discover

$$
\lambda=h\left(0_{x(s)} \oplus \dot{\bar{x}}\left(t_{0}\right), \dot{x}(s) \oplus 0_{\bar{x}\left(t_{0}\right)}\right)=-\dot{x}^{\dot{i}}\left(t_{0}\right) \dot{q}_{\bar{i}}^{*}(s) .
$$

Integrating this constant over $0<s<1,4.12$ yields the desired orthogonality

$$
\lambda=\dot{x}^{\bar{i}}\left(t_{0}\right)\left(q_{i}^{*}(0)-q_{\dot{i}}^{*}(1)\right)=0 .
$$

Now 4.13 shows $\partial^{2} g /\left.\partial t^{2}\right|_{t=t_{0}}$ to be a convex function of $s \in[0,1]$. We shall prove this convex function is minimized at $s=0$, where it vanishes. Introducing coordinates $x^{1}, \ldots, x^{n}$ around $x=x(0)$ on $M$ and $x^{\overline{1}}, \ldots, x^{\bar{n}}$ around $\bar{x}\left(t_{0}\right)$ on $\bar{M}$, we compute

$$
\begin{aligned}
\left.\frac{\partial^{2} g}{\partial t^{2}}\right|_{\left(s, t_{0}\right)} & =-\left[c_{\bar{i}}^{-}(x(s), \bar{x}(t)) \ddot{x^{i}}+c_{\bar{i} \bar{j}}^{-}(x(s), \bar{x}(t)) \dot{x}^{\bar{i}} \dot{x}^{\bar{j}}\right]_{\left(0, t_{0}\right)}^{\left(s, t_{0}\right)}, \\
\left.\frac{\partial^{3} g}{\partial s \partial t^{2}}\right|_{\left(s, t_{0}\right)} & =-\left(c_{\bar{i} k}^{-}\left(x(s), \bar{x}\left(t_{0}\right)\right) \ddot{x}^{\bar{i}}+c_{\bar{i} \bar{j} k}^{-}\left(x(s), \bar{x}\left(t_{0}\right)\right) \dot{x}^{\bar{i}} \dot{x}^{\bar{j}}\right) \dot{x}^{k} .
\end{aligned}
$$

When $s=0$, the last line vanishes by the geodesic equation for $t \in[0,1] \mapsto(x(0), \bar{x}(t))$, and the preceding line is manifestly zero. Thus the strictly convex function $\partial^{2} g /\left.\partial t^{2}\right|_{t=t_{0}}$ must be non-negative for $s \in[0,1]$ and, as initially claimed, $\ddot{f}\left(t_{0}\right)=\partial^{2} g /\left.\partial t^{2}\right|_{(s, t)=\left(1, t_{0}\right)}$ is non-negative at any $\left.t_{0} \in\right] 0,1\left[\right.$ where $\dot{f}\left(t_{0}\right)=0$.

If the relevant cross-curvature of the cost is positive at one point $\left(x\left(s_{0}\right), \bar{x}\left(t_{0}\right)\right)$, then the non-negative function $\partial^{2} g /\left.\partial t^{2}\right|_{t=t_{0}}$ is strictly convex 4.13) on an interval around $s_{0} \in[0,1]$; since $\partial^{2} g /\left.\partial t^{2}\right|_{t=t_{0}}$ is minimized at $s=0$ it must then be positive at $s=1$, to conclude the proof. 
Definition 4.7 (Illuminated set). Given $(x, \bar{x}) \in N$, let $V(x, \bar{x}) \subset M$ denote those points $y \in N(\bar{x})$ for which there exists a curve from $(x, \bar{x})$ to $(y, \bar{x})$ in $N(\bar{x}) \times\{\bar{x}\}$ satisfying the geodesic equation on $(N, h)$.

As a corollary to Proposition 4.6 and warm up to the double mountain above sliding mountain Theorem 4.10 let us derive a strong version of this result under the simplifying hypothesis that the cost is strictly (A3s) and not merely weakly regular (A3w).

Corollary 4.8 (Strict maximum principle). Use a strictly regular cost $c \in C^{4}(N)$ to define a pseudo-metric 2.1) on the domain $N \subset M \times \bar{M}$. Let $t \in[0,1] \mapsto(x, \bar{x}(t)) \in N$ be a geodesic in $(N, h)$ with $\dot{\bar{x}}(0) \neq 0$. Then for all $y \in \bigcap_{t \in[0,1]} V(x, \bar{x}(t))$ the function $f(t)=-c(y, \bar{x}(t))+c(x, \bar{x}(t))$ satisfies $f(t)<\max \{f(0), f(1)\}$ on $0<t<1$.

Proof. Given $y \in \bigcap_{t \in[0,1]} V(x, \bar{x}(t))$ we have $y, x \in N(\bar{x}(t))$ so $f$ is $C^{4}$ smooth on $[0,1]$. Proposition 4.6 asserts $\ddot{f}\left(t_{0}\right)>0$ at each interior critical point $\dot{f}\left(t_{0}\right)=0$. Any critical point of $f$ in ]0, 1 [ is therefore a local minimum, and $f$ is strictly monotone away from this point. Thus $f(t)<\max \{f(0), f(1)\}$ for $0<t<1$, as desired.

If $N$ is horizontally convex then $V(x, \bar{x}(t))=N(\bar{x}(t))$, which motivates the relation of this corollary to Theorem 3.1. Let us now show a weak version of this maximum principle survives as long as the cost is weakly regular. To handle this relaxation we use a level set approach.

Lemma 4.9 (Level set evolution). Let $g \in C^{2}(] \epsilon, \epsilon[\times U)$ where $U \subset \mathbb{R}^{n}$ is open. Suppose $D g=\left(\partial_{1} g, \ldots, \partial_{n} g\right)$ is non-vanishing on $] \epsilon, \epsilon[\times U$. Then the zero set $S(t)=$ $\{x \in U \mid g(t, x)=0\}$ is a $C^{2}$-smooth $(n-1)$-dimensional submanifold of $U$ which can be parameterized locally for small enough t by $\{X(t, z) \mid z \in S(0)\}$, where the Lagrangian variable $X(t, x)$ solves the ordinary differential equation

$$
\frac{\partial X(t, x)}{\partial t}=-\left[\frac{\partial g}{\partial t} \frac{D g}{|D g|^{2}}\right]_{(t, X(t, x))}
$$

subject to the initial condition $X(t, x)=x$. Moreover, the positivity set $S^{+}(t)=\{x \in U \mid$ $g(t, x) \geq 0\}$ has $S(t)$ as its boundary, and expands with an outward normal velocity given by

$$
v=-\left.\left.\frac{\partial X}{\partial t}\right|_{(t, z)} \cdot \frac{D g}{|D g|}\right|_{(t, X(t, z))}=\left.\frac{\partial g / \partial t}{|D g|}\right|_{(t, X(t, z))} .
$$

Proof. Clearly the boundary of $S^{+}(t)$ is contained in $S(t)$. Since $D g \neq 0$, the implicit function theorem implies $S(t)$ is a $C^{2}$-smooth hypersurface and separates regions where $g(t, x)$ takes opposite signs. Thus $S(t)$ is contained in and hence equal to the boundary in $U$ of the positivity set. If the desired parameterization exists it must satisfy $0=g(t, X(t, z))$. Differentiation in time yields an equation

$$
0=\frac{\partial g}{\partial t}(t, X(t, z))+\left.D g(t, X(t, z)) \cdot \frac{\partial X}{\partial t}\right|_{(t, z)},
$$


easily seen to be equivalent to (4.14). Conversely, near a point $(0, z) \in]-\epsilon, \epsilon[\times S(0)$, the $C^{1}$ vector field 4.14 can be integrated for a short time (depending on $z$ ) to yield the desired parameterization.

Theorem 4.10 (Double mountain above sliding mountain). Use a weakly regular cost $c \in C^{4}(N)$ to define a pseudo-metric $\sqrt{2.1}$ on the domain $N \subset M \times \bar{M}$. Let $\left.t \in\right] 0,1[\mapsto$ $(x, \bar{x}(t)) \in N$ be a geodesic in $(N, h)$. If $] t_{0}, t_{1}[\times\{y\}$ lies in the interior of

$$
\Lambda:=\{(t, y) \in[0,1] \times M \mid y \in V(x, \bar{x}(t))\},
$$

then $f(t)=-c(y, \bar{x}(t))+c(x, \bar{x}(t)) \leq \max \left\{f\left(t_{0}^{+}\right), f\left(t_{1}^{-}\right)\right\}$on $0 \leq t_{0}<t<t_{1} \leq 1$, where

$$
f\left(t_{0}^{+}\right)=\lim _{\epsilon \searrow 0} f\left(t_{0}+\epsilon\right), \quad f\left(t_{1}^{-}\right)=\lim _{\epsilon \searrow 0} f(t-\epsilon) .
$$

Proof. Fix a geodesic $t \in] 0,1[\mapsto(x, \bar{x}(t)) \in N$ with $\dot{\bar{x}}(1 / 2) \neq 0$, since otherwise the conclusion is obvious. Note that $c$ - $\operatorname{Exp}_{x}$ and hence $\left.t \in\right] 0,1\left[\mapsto \bar{x}(t)\right.$ are $C^{3}$ smooth, from Lemma 4.4 and (A0). For all $y \in M$ and $t \in] 0,1[$ set

$$
f(t, y)=-c(y, \bar{x}(t))+c(x, \bar{x}(t))
$$

and note that $f(t, y)$ is $C^{3}$-smooth on the interior of $\Lambda \subset[0,1] \times M$. Define

$$
\begin{aligned}
S^{+} & :=\left\{(t, y) \in \operatorname{int} \Lambda \mid \frac{\partial f}{\partial t} \geq 0\right\}, \\
S & :=\left\{(t, y) \in \operatorname{int} \Lambda \mid \frac{\partial f}{\partial t}=0\right\} .
\end{aligned}
$$

For each $t \in] 0,1$, we think of $f(t, y)$ as defining the elevation of a landscape over $M$, which evolves from $f(y, 0)$ to $f(y, 1)$ as $t$ increases, and is normalized so that $f(x, t)$ $=0$. We picture $f(y, t)$ as a sliding mountain, with $S^{+}(t):=\left\{y \in M \mid(t, y) \in S^{+}\right\}$ denoting the rising region, and $S(t):=\{y \in M \mid(t, y) \in S\}$ the region at the boundary of $S^{+}(t)$ which—instantaneously—is neither rising nor sinking.

We claim the rising region $S^{+}(t) \subset M$ is a non-decreasing function of $\left.t \in\right] t_{0}, t_{1}[$. To see this, we plan to apply Lemma 4.9 to the $C^{2}$ function

$$
g(t, y):=\frac{\partial f}{\partial t}=-\bar{D} c(y, \bar{x}(t)) \dot{\bar{x}}(t)+\bar{D} c(x, \bar{x}(t)) \dot{\bar{x}}(t)
$$

on $\Lambda$. Differentiating this function with respect to $y \in M$ yields

$$
D g(t, y)=-D \bar{D} c(y, \bar{x}(t)) \dot{\bar{x}}(t) \neq 0
$$

because of (A2). Applying Lemma 4.9 on any coordinate chart in $M$ shows $S(t)$ is the boundary of $S^{+}(t)$, and the question of whether $S^{+}(t)$ is expanding or contracting along its boundary is determined by the sign of $\partial g / \partial t$ on $S(t)$. 
From the definition of $V\left(x, \bar{x}\left(t^{\prime}\right)\right)$ observe $y \in S\left(t^{\prime}\right) \subset V\left(x, \bar{x}\left(t^{\prime}\right)\right)$ implies $\left(y, \bar{x}\left(t^{\prime}\right)\right)$ is linked to $\left(x, \bar{x}\left(t^{\prime}\right)\right)$ by a curve in $N\left(\bar{x}\left(t^{\prime}\right)\right) \times\left\{\bar{x}\left(t^{\prime}\right)\right\}$ which is geodesic in $(N, h)$. Proposition 4.6 asserts $\partial^{2} f /\left.\partial t^{2}\right|_{\left(t^{\prime}, y\right)} \geq 0$, so from Lemma 4.9 there is a neighbourhood of $y$ on which the rising region $S^{+}(t)$ does not shrink for a short time interval around $\left.t^{\prime} \in\right] 0,1[$.

Finally, fix $y \in M$ and $0 \leq t_{0}<t_{1} \leq 1$ such that $] t_{0}, t_{1}[\times\{y\} \subset$ int $\Lambda$ and let $U \subset] t_{0}, t_{1}$ [ denote the open set of times at which $y \notin S^{+}(t)$. If $U$ is non-empty, we claim any connected component of $U$ has $t=t_{0}$ in its closure. If not, let $\left.t^{\prime} \in\right] t_{0}, t_{1}[$ denote the left endpoint of a connected component in $U$. This means $y \in S^{+}\left(t^{\prime}\right)$ but $y \notin S^{+}\left(t^{\prime}+\delta\right)$ for any $\delta>0$, in violation of the non-shrinking property of $S^{+}(t)$ derived above. Thus $t \in] t_{0}, t_{1}[\mapsto f(t, y)$ is decreasing on an interval $U=] t_{0}, t(y)$ [ for some $t(y) \in\left[t_{0}, t_{1}\right]$ and non-decreasing on the complementary interval $] t(y), t_{1}[$. The limits 4.17 exist and the proof that $f(t, y) \leq \max \left\{f\left(t_{0}^{+}\right), f\left(t_{1}^{-}\right)\right\}$is complete.

Remark 4.11 (A geodesic hypersurface bounds the rising region). Lemma 4.4 implies $S(t) \times\{\bar{x}(t)\}$ is a totally geodesic submanifold for each $t \in$ ]0,1[ of the preceding proof. Indeed, 4.18 shows $\bar{q}^{*} \in \operatorname{Dom}\left(\check{c}\right.$-Exp $\left.\bar{x}_{\bar{x}(t)}\right) \subset T_{\bar{x}(t)}^{*} \bar{M}$ belongs to the hyperplane $\left(\bar{D} c(x, \bar{x}(t))+\bar{q}^{*}\right) \bar{x}(t)=0$ if and only if $y:=\check{c}$-Exp $\bar{x}_{(t)} \bar{q}^{*}$ lies on $S(t)$.

Remark 4.12. If the domain $N$ is horizontally convex then $V(x, \bar{x})=N(\bar{x})$ and $\Lambda$ from 4.16 are open sets. Then $] 0,1\left[\times\{y\} \in \Lambda\right.$ if and only if $y \in \bigcap_{0<t<1} N(\bar{x}(t))$.

Remark 4.13 (Enhancements and further developments). At the time of our original submission, Villani had incorporated a version of our proof into [50], modifying it to allow him to avoid the use of the level set method. He further developed our technique to prove stronger results: contrast Theorem 3.1 above (and the first theorem in [27]) with Villani's Theorem 12.36, which extends Loeper's maximum principle to new Riemannian manifolds. Since our original submission, many further developments have appeared, due to Figalli \& ourselves [17], Figalli \& Loeper [18], Figalli \& Rifford [19], Figalli \& Villani [20], Loeper \& Villani [33], Trudinger \& Wang [41], Villani [48], and ourselves [29], stimulated in part by the present manuscript and its precursor [28]. These include Hölder continuity results for maps between rough measures which optimize weakly regular costs in two dimensions [18] and continuity results for such maps for non-negatively crosscurved costs in higher dimensions [17], regularity of optimal maps on strictly regular Riemannian manifolds with no purely-focal points [33], stability of the strong regularity hypothesis [48], and regularity results for optimal maps with respect to perturbations of the spherical metric [19].

\section{Perspective and conclusions}

Before concluding this paper, let us briefly review the connection of the transportation problem 1.1 we study with fully non-linear partial differential equations. Although this connection goes almost back to Monge [38], it has developed dramatically since the work of Brenier [3]. When an optimal mapping $F: M \rightarrow \bar{M}$ exists and happens to be a 
diffeomorphism, it provides a change of variables between $(M, \rho)$ and $(\bar{M}, \bar{\rho})$, hence its Jacobian satisfies the equation

$$
\bar{\rho}(F(x))|\operatorname{det} D F(x)|=\rho(x) .
$$

Often when $F$ is not smooth, (5.1) remains true almost everywhere [35], [12], [1].

Optimality implies that $F(\cdot)$ can be related to a pair of scalar functions $u: M \rightarrow$ $\mathbb{R} \cup\{+\infty\}$ and $\bar{u}: \bar{M} \rightarrow \mathbb{R} \cup\{+\infty\}$, which arise from the linear program dual to 1.1 , and represent Lagrange multipliers for the prescribed densities $\rho$ and $\bar{\rho}$. Moreover, $u$ can be taken to belong to the class of $c$-convex functions, meaning it can be obtained as the upper envelope of a family of mountains - analogous to $(3.1)$ and (3.8) but with $i$ ranging over $\bar{\rho}$-almost all of $\bar{M}$; similarly, $\bar{u}$ is a $\check{c}$-convex function, meaning $\bar{u}=\left(\bar{u}^{c}\right)^{c}$ in A.1 (see Appendix). When the twist condition holds, $F=c$-Exp $\circ D u$. If non-degeneracy (A2) also holds, then (5.1) becomes an equation of Monge-Ampère type expressed as in [34] using local coordinates around $\left(x^{\prime}, F\left(\bar{x}^{\prime}\right)\right)$ by

$$
\operatorname{det}\left[u_{i j}+c_{i j}\right]_{\left(x, c-\operatorname{Exp}_{x} D u(x)\right)}=\frac{\rho(x)}{\bar{\rho}\left(c-\operatorname{Exp}_{x} D u(x)\right)}\left|\operatorname{det} c_{i \bar{k}}\right|_{\left(x, c-\operatorname{Exp}_{x} D u(x)\right)} ;
$$

$c$-convexity of $u$ implies non-negative definiteness of the matrix $u_{i j}+c_{i j}$ of second derivatives, hence the equation is degenerate elliptic [22]. Without further assumptions on $(M, \rho)$ and the geometry of $(\bar{M}, \bar{\rho})$ we can expect neither strict ellipticity nor regularity of solutions: if the support of $\rho$ is connected but the support of $\bar{\rho}$ is not, this will force $F$ to be discontinuous and $u \notin C^{1}(M)$. The equation is therefore not locally smoothing, and the best one can hope is for solutions to inherit regularity from the boundary data $(M, \rho)$ and $(\bar{M}, \bar{\rho})$.

For the cost function $c(x, \bar{x})=|x-\bar{x}|^{2} / 2$ on $M, \bar{M} \subset \mathbb{R}^{n}$, 5 5.2) becomes the familiar Monge-Ampère equation for the convex function $u(x)+|x|^{2} / 2[3]$. In this case Caffarelli [5] was able to show convexity of $\bar{M}$ implies local Hölder continuity of $F$ if the densities $d \rho / d \mathrm{vol} \in L^{\infty}(M)$ and $d \mathrm{vol} / d \bar{\rho} \in L^{\infty}(\bar{M})$ are bounded, and smoothness of $F$ on the interior of $M$ if the densities $\log |d \rho / d \mathrm{vol}|$ and $\log |d \bar{\rho} / d \mathrm{vol}|$ are bounded and smooth; see also Delanoë [13] for the case $n=2$, and Wang [52] for analogous results in the context of Example 3.5. the reflector antenna problem. The results of Loeper and Ma, Trudinger \& Wang extend the Hölder [31] and smooth [34], [43] theories to general bi-twisted, strictly regular costs on $\operatorname{cl}(M \times \bar{M})$. Loeper in particular achieves stronger results such as a global Hölder estimate with explicit exponent under weaker restrictions on $\rho$ and $\bar{\rho}$ by exploiting strict regularity of the cost [31]. Trudinger \& Wang extended the up-to-the-boundary regularity results of Caffarelli [4] and Urbas [44]—which require convexity and smoothness of $M \subset \mathbb{R}^{n}$ as well as $\bar{M}$ - to bi-twisted costs which are merely weakly regular [42]. For bounded and sufficiently smooth densities, horizontal and vertical convexity of $N=M \times \bar{M}$ takes the place of the convexity assumptions on the target $\bar{M}$ and domain $M$ in these theories.

Since we had not previously encountered pseudo-Riemannian geometry of any signature other than the Lorentzian one $(n, 1)$ in applications, much less as the necessary and 
sufficient condition for degenerate elliptic partial differential equations to possess smooth solutions, a few words of explanation seem appropriate 2

The regularity of optimal maps $F: M \rightarrow \bar{M}$ is a question whose answer should depend only on the cost function $c(x, \bar{x})$ and the probability measures $\rho$ and $\bar{\rho}$. It should not depend on which choice of smooth coordinates on $M$ and $\bar{M}$ are used to represent this data or the solution. Any necessary and sufficient condition on $c(x, \bar{x})$ guaranteeing regularity of $F$ should therefore be geometrically invariant, meaning coordinate independent. Thus pseudo-Riemannian geometry and curvatures arise in the theory of optimal transportation for the same reason they arise in Einstein's theory of gravity, general relativity: they provide the natural language for describing phenomena-in this case regularitywhich exhibit invariance under the general group of diffeomorphisms. Put another way, the underlying physical reality is independent of which coordinates are used to describe it. Moreover, this invariance places severe restrictions on the form which necessary and sufficient conditions for regularity can take: since the pseudo-metric $h$ is equivalent to knowing the cost function $c(x, \bar{x})$ - up to null Lagrangians $v(x)+\bar{v}(\bar{x})$ which are irrelevant to the optimization at hand $(1.1)$ - any such conditions on the cost function must be expressible via the curvature tensor of $h$.

Let us now turn to the question of why the intrinsic geometry of optimal transportation should be pseudo-Riemannian rather than Riemannian. Dimensional symmetry between the domain $M$ and target $\bar{M}$ suggests that the number of time-like directions in the theory - if any — should equal the number of space-like directions. But why signature $(n, n)$ rather than the signature $(2 n, 0)$, which is more frequently associated to elliptic and extremal problems? And why the nullity of $p \oplus \bar{p}$ in (A3w)?

The Riemannian notion of length allows us to associate a magnitude to any sectional curvature. However, null vectors have no length and cannot be normalized; because the plane $(p \oplus 0) \wedge(0 \oplus \bar{p})$ is generated by orthogonal null vectors, we can decide the sign (positive, negative, or zero) of its sectional curvature, but not the magnitude. On the other hand, the results of Loeper reveal that the size of the constant $C>0$ in hypothesis 1.2 controls the Hölder constant of the mapping $F: M \rightarrow \bar{M}$. Unlike the exponent, which is coordinate-independent, the Hölder constant of $F$ obviously depends on the choice of coordinates. We are therefore relieved to find the cross-curvature condition governing regularity does not have an associated magnitude, since the problem has no intrinsic length scale. To be scale free, the geometrical structure which governs regularity for optimal transportation must be pseudo-Riemannian, since the modulus of continuity of a map $F$ has no intrinsic meaning in the absence of separate notions of length on $M$ and $\bar{M}$, which the cost function $c(x, \bar{x})$ alone cannot provide. What it can and does

2 However, as we learned subsequently from Robert Bryant, the wedge product $\omega \wedge \omega$ can be used to define a signature $(3,3)$ pseudo-metric on the space $\mathbb{R}^{4} \wedge \mathbb{R}^{4}$; in four dimensions, the difference between positive sectional curvature and positive curvature operator amounts to the question of whether the curvature operator is positive definite only on the light cone with respect to this pseudo-metric, or on the full space (cf. [2]). In one way this parallels the distinction between strict regularity and positive cross-curvature of a cost; in another it parallels the distinction between positive cross-curvature and positive sectional curvature, 2.3 - 2.4 . 
provide are geodesics on $N(\bar{x}) \times\{\bar{x}\}$ and $\{x\} \times N(x)$, and geodesic convexity of these null submanifolds is the essential domain hypothesis in Ma, Trudinger \& Wang's theory.

In the main text of this manuscript, we have focused exclusively on the pseudo-metric $d \ell^{2}=-c_{i} \bar{j} d x^{i} d x^{\bar{j}}$ induced by the cost 2.1 . Let us conclude by noting that there is also a canonical symplectic form $\omega=d(D c \oplus 0)=-d(0 \oplus \bar{D} c)$ on $N \subset M \times \bar{M}$ associated to the cost $c \in C^{4}(N)$. In local coordinates $x^{1}, \ldots, x^{n}$ on $M$ and $x^{\overline{1}}, \ldots, x^{\bar{n}}$ on $\bar{M}$ it is given by

$$
\omega:=\frac{1}{2}\left(\begin{array}{cc}
0 & \bar{D} D c \\
-D \bar{D} c & 0
\end{array}\right) .
$$

It is possible to verify that any $c$-optimal diffeomorphism $F: M \rightarrow \bar{M}$ has a graph which is spacelike with respect to $h$ and Lagrangian with respect to $\omega$. Conversely, for a weakly regular cost, results of Trudinger \& Wang [42], [43] can be used to deduce that any diffeomorphism whose graph is $h$-spacelike and $\omega$-Lagrangian is in fact the $c$-optimal map between the measures $\rho:=\pi_{\#}\left(\left.\operatorname{vol}^{(N, h)}\right|_{\operatorname{Graph}(F)}\right)$ and $\bar{\rho}:=\bar{\pi}_{\#}\left(\left.\operatorname{vol}^{(N, h)}\right|_{\operatorname{Graph}(F)}\right)$ obtained by projecting the Riemannian volume $\operatorname{vol}^{(N, h)}$ induced by $h$ on $\operatorname{Graph}(F)$ through the canonical projections $\pi(x, \bar{x})=x$ and $\bar{\pi}(x, \bar{x})=\bar{x}$. This reveals another unexpected connection between optimal transportation and symplectic (or para-Kähler) geometry. When $\rho$ and $\bar{\rho}$ are given by the Euclidean volumes on two convex domains, and $c(x, \bar{x})=|x-\bar{x}|^{2} / 2$, this is related to the work of Wolfson [55] and Warren [54] on special Lagrangian submanifolds, where a pseudo-Riemannian metric of signature $(n, n)$ also appears [54]. We defer the details of this development to the appendix below.

\section{Appendix. Differential geometric characterization of optimality}

This appendix is intended to elucidate how differential geometric concepts such as Lagrangian submanifolds describe optimizers in the Kantorovich problem (1.1). It was added in revision at the request of the referee, to substantiate claims made in the concluding remarks of the text. For simplicity, we shall focus our attention particularly on situations in which the optimizers are known to be supported on the graph of a differentiable map $F: M \rightarrow \bar{M}$.

For Lipschitz costs, it is well-known that $\gamma \in \Gamma(\rho, \bar{\rho})$ minimizes 1.1 if and only if $\gamma$ vanishes outside a set $\Sigma \subset M \times \bar{M}$ which is $c$-cyclically monotone [22], [49]. In turn, $\Sigma$ is $c$-cyclically monotone if and only if there exists a pair of Lipschitz functions $u \in C(\operatorname{cl} M)$ and $\bar{u} \in C(\operatorname{cl} \bar{M})$ which are conjugate to each other in the sense that $u=\bar{u}^{c}$ and $\bar{u}=u^{\check{c}}$, where

$$
\bar{u}^{c}(x)=\sup _{\bar{x} \in \operatorname{cl} \bar{M}}(-c(x, \bar{x})-\bar{u}(\bar{x})), \quad u^{\check{c}}(\bar{x})=\sup _{x \in \operatorname{cl} M}(-c(x, \bar{x})-u(x)),
$$

and for which $\Sigma \subset \partial^{c} u$ with $\partial^{c} u=\partial_{\mathrm{cl}}^{c} M^{u}$ from 2.8. We may take such an inclusion to define $c$-cyclical monotonicity in the present context. In case $\Sigma$ is a smooth graph, the next theorem uses these facts to characterize optimality in terms of the pseudo-metric $h$ and symplectic form $\omega$ induced by the cost through 2.1. and 5.3. The second part of 
the theorem should be compared to a result of Trudinger and Wang giving local sufficient conditions on $u$ for $c$-convexity: $u=u^{\breve{c} c}$ [43]; see also [42], [41] and Villani's Theorem 12.46 [50]. Although part (2) of Theorem A.1] can be deduced from their result, the argument given below is based on considerations introduced above and entirely self-contained; at least for $u \in C^{2}(M)$, it yields a new, logically independent, proof of Trudinger and Wang's result, without requiring twistedness of the cost.

Recall a submanifold $\Sigma \subset N$ is Lagrangian with respect to the symplectic form $\omega$ if $\omega(P, Q)=0$ for all $s \in \Sigma$ and tangent vectors $P, Q \in T_{s} \Sigma$. The same submanifold is spacelike with respect to the pseudo-metric $h$ if $h(P, P) \geq 0$ for all $s \in \Sigma$ and tangent vectors $P \in T_{S} \Sigma$, and strictly spacelike if equality implies $P=0$. The next theorem shows that being $\omega$-Lagrangian and $h$-spacelike give local differential conditions on a graph which characterize global minimality. This is somehow analogous to the wellknown result for functions on $\mathbb{R}^{n}$, that a (local) minimum has vanishing first derivative and non-negative second derivative. The converse may also be true, but only if the second derivative is strictly positive or if some global information about the function whose critical points are being investigated is known — such as convexity, unimodality, or level set convexity - which allows global minimality to be inferred from local criticality. In part (2) of the theorem below, Ma, Trudinger and Wang's hypotheses on the domains and cost provides this requisite global information.

Theorem A.1 (Spacelike Lagrangian characterization of optimality). Fix $c \in C^{4}(N)$ non-degenerate on the domain $N \subset M \times \bar{M}$ extending to a Lipschitz function on the compact space $\operatorname{cl}(M \times \bar{M})$. Let $F: M \rightarrow \bar{M}$ be differentiable.

(1) If $\operatorname{Graph}(F):=\{(x, F(x)) \mid x \in M\}$ is c-cyclically monotone, then $\operatorname{Graph}(F) \cap N$ is Lagrangian for the symplectic form $\omega$ of 5.3 , and spacelike for the pseudo-metric $h$ of (2.1); it is strictly spacelike at points where $\operatorname{det}[D F(x)] \neq 0$.

(2) Conversely, assuming $c$ is weakly regular and $N=M \times \bar{M}$ is bi-convex, if $\operatorname{Graph}(F)$ is $\omega$-Lagrangian and h-spacelike then $\operatorname{Graph}(F)$ is c-cyclically monotone.

Proof of (1). Suppose $\Sigma=\operatorname{Graph}(F)$ is $c$-cyclically monotone. As asserted above, this means $\Sigma \subset \partial^{c} u$ for a Lipschitz function $u \in C(\mathrm{cl} M)$. Assuming differentiability of $F$, we shall now deduce $u \in C^{2}$, at least on the projection $U=\pi^{M}(\Sigma \cap N)$. Since $(x, F(x)) \in \partial^{c} u$ implies $y \in M \mapsto u(y)+c(y, F(x))$ is minimized at $y=x$, we have

$$
D u(x)=-D c(x, F(x))
$$

on the set $x \in \operatorname{Dom} D u$ of full measure in $U$. Here the right hand side is continuously differentiable on $U$, implying $u \in C^{2}(U)$. Denoting $F_{j}^{\bar{k}}:=\partial F^{\bar{k}} / \partial x^{j}$ and differentiating A.2 yields symmetry of the matrix

$$
u_{i j}+c_{i j}=-c_{i \bar{k}} F_{j}^{\bar{k}} \geq 0
$$

in any local coordinate system $x^{1}, \ldots, x^{n}$ on $M$ and $x^{\overline{1}}, \ldots, x^{\bar{n}}$ on $\bar{M}$. This matrix is non-negative definite since $y \in M \mapsto u(y)+c(y, F(x))$ attains its minimum at $y=x$. 
The non-degeneracy of $c$ shows the same matrix to be invertible, hence positive definite, wherever $\operatorname{det}\left[D F\left(x_{0}\right)\right] \neq 0$.

Any pair of smooth curves intersecting on $N \cap \operatorname{Graph}(F)$ can be parameterized near their point of intersection $\left(x_{0}, F\left(x_{0}\right)\right)$ by $s \in[-1,1] \mapsto(x(s), F(x(s)))$ and $t \in[-1,1]$ $\mapsto(y(t), F(y(t)))$ with $x(0)=y(0)=x_{0} \in U$. Setting $P=\left[I \mid D F\left(x_{0}\right)\right] \dot{x}(0)$ and $Q=\left[I \mid D F\left(x_{0}\right)\right] \dot{y}(0)$, from $\mathrm{A} .3$ we compute

$$
2 \omega(P, Q):=\dot{x}^{i} c_{i \bar{j}} F_{k}^{\bar{j}} \dot{y}^{k}-\dot{x}^{i} F_{i}^{\bar{j}} c_{\bar{j} k} \dot{y}^{k}=-\dot{x}^{i}\left(u_{i k}+c_{i k}\right) \dot{y}^{k}+\dot{x}^{i}\left(u_{k i}+c_{k i}\right) \dot{y}^{k}=0
$$

and

$$
2 h(P, P):=-\dot{x}^{i} c_{i j} F_{k}^{\bar{j}} \dot{x}^{k}-\dot{x}^{i} F_{i}^{\bar{j}} c_{j k} \dot{x}^{k}=\dot{x}^{i}\left(u_{i k}+c_{i k}\right) \dot{x}^{k}+\dot{x}^{i}\left(u_{k i}+c_{k i}\right) \dot{x}^{k} \geq 0 .
$$

In other words, $\Sigma$ is $\omega$-Lagrangian and $h$-spacelike in $N$, and strictly spacelike wherever $\operatorname{det}\left[D F\left(x_{0}\right)\right] \neq 0$.

Proof of (2). To prove the converse, assume $N=M \times \bar{M}$ bi-convex and $\Sigma=\operatorname{Graph}(F)$ is $\omega$-Lagrangian and $h$-spacelike. In local coordinates, this means the matrix $-c_{i} \bar{j} F_{k}^{\bar{j}}$ is symmetric and non-negative definite. Symmetry implies the field of covectors $-D c(x, F(x))$ agrees with a differential $D u(x)$ of $u \in C^{2}(M)$ locally, and in fact globally due to the Poincaré lemma since bi-convexity of $N$ implies contractibility of $M$. Note $u$ extends to a Lipschitz function on $\mathrm{cl} M$ since $c$ is Lipschitz on $\operatorname{cl}(M \times \bar{M})$. Non-negative definiteness implies $u_{i j}+c_{i j} \geq 0$. We use this in the following, to show that $u$ is $c$-convex, meaning $u=\left(u^{\check{c}}\right)^{c}$ in A.1. This $c$-convexity implies the desired $c$-cyclical monotonicity of $\operatorname{Graph}(F)$.

Fix $x_{0} \in M$ and $x \in \operatorname{cl} M$. Let $\bar{x}_{0}=F\left(x_{0}\right)$. It suffices to show that

$$
u(x)-u\left(x_{0}\right)+c\left(x, \bar{x}_{0}\right)-c\left(x_{0}, \bar{x}_{0}\right) \geq 0 .
$$

This implies $-u\left(x_{0}\right)-c\left(x_{0}, \bar{x}_{0}\right)=u^{\check{c}}\left(\bar{x}_{0}\right)$ and hence $u^{\check{c} c}\left(x_{0}\right) \geq u\left(x_{0}\right)$. The converse inequality $u \geq u^{\check{c} c}$ holds quite generally [49]. Moreover, since $u$ is Lipschitz it suffices to establish $u^{\check{c} c} \geq u$ and A.4 for all $x, x_{0} \in M$ to conclude the desired identity $u^{\breve{c} c}=u$ on cl $M$. We apply the same idea as in Theorem 4.10. We first show

Claim A.2. Let $t \in[0,1] \mapsto(x(t), \bar{x}) \in N$ be a horizontal geodesic with $x_{0}=x(0)$ and $x=x(1)$. Define

$$
H(t, \bar{x})=u(x(t))+c(x(t), \bar{x}) .
$$

If $\frac{\partial}{\partial t} H(t, \bar{x})=0$, then $\frac{\partial^{2}}{\partial t^{2}} H(t, \bar{x}) \geq 0$.

Proof of Claim A.2. Fix $\bar{x}$ and suppose $\left.\frac{\partial}{\partial t}\right|_{t=t_{0}} H(t, \bar{x})=0$. Let $\bar{x}_{t_{0}}=F\left(x\left(t_{0}\right)\right)$; that is, $D u\left(x\left(t_{0}\right)\right)+D c\left(x\left(t_{0}\right), \bar{x}_{t_{0}}\right)=0$, and

$$
\begin{aligned}
\left.\frac{\partial^{2}}{\partial t^{2}}\right|_{t=t_{0}} H\left(t, \bar{x}_{t_{0}}\right)= & \left(u_{i j}\left(x\left(t_{0}\right)\right)+c_{i j}\left(x\left(t_{0}\right), \bar{x}_{t_{0}}\right)\right) \dot{x}\left(t_{0}\right)^{i} \dot{x}\left(t_{0}\right)^{j} \\
& +\left(u_{i}\left(x\left(t_{0}\right)\right)+c_{i}\left(x\left(t_{0}\right), \bar{x}_{t_{0}}\right)\right) \ddot{x}\left(t_{0}\right)^{i} \\
= & \left(u_{i j}\left(x\left(t_{0}\right)\right)+c_{i j}\left(x\left(t_{0}\right), \bar{x}_{t_{0}}\right)\right) \dot{x}\left(t_{0}\right)^{i} \dot{x}\left(t_{0}\right)^{j} \\
\geq & 0 .
\end{aligned}
$$


The last line follows from the non-negative definiteness of $u_{i j}+c_{i j} \geq 0$ on $\operatorname{Graph}(F)$. Consider a vertical geodesic $s \in[0,1] \mapsto\left(x\left(t_{0}\right), \bar{x}(s)\right)$ linking $\bar{x}(0)=\bar{x}_{t_{0}}$ to $\bar{x}(1)=\bar{x}$; it satisfies

$$
c_{i} \bar{j}\left(x\left(t_{0}\right), \bar{x}(s)\right) \dot{\bar{x}}(s)^{\bar{j}}=-c_{i}\left(x\left(t_{0}\right), \bar{x}_{t_{0}}\right)+c_{i}\left(x\left(t_{0}\right), \bar{x}\right) .
$$

Therefore,

$$
\begin{aligned}
0 & =\left.\frac{\partial}{\partial t}\right|_{t=t_{0}} H(t, \bar{x})=u_{i}\left(x\left(t_{0}\right)\right) \dot{x}\left(t_{0}\right)^{i}+c_{i}\left(x\left(t_{0}\right), \bar{x}\right) \dot{x}\left(t_{0}\right)^{i} \\
& =-c_{i}\left(x\left(t_{0}\right), \bar{x}_{t_{0}}\right) \dot{x}\left(t_{0}\right)^{i}+c_{i}\left(x\left(t_{0}\right), \bar{x}\right) \dot{x}\left(t_{0}\right)^{i} \\
& =c_{i} \bar{j}\left(x\left(t_{0}\right), \bar{x}(s)\right) \dot{x}\left(t_{0}\right)^{i} \dot{\bar{x}}(s)^{\bar{j}}
\end{aligned}
$$

Thus we can use weak regularity $(\mathbf{A} 3 \mathbf{w})$ of the cost in Lemma 4.5 to deduce

$$
\left.\frac{\partial^{4}}{\partial s^{2} \partial t^{2}}\right|_{(s, t)=\left(s, t_{0}\right)} H(t, \bar{x}(s))=\left.\frac{\partial^{4}}{\partial s^{2} \partial t^{2}}\right|_{(s, t)=\left(s, t_{0}\right)} c(x(t), \bar{x}(s)) \leq 0 .
$$

From the geodesic equation satisfied by $t \in[0,1] \mapsto(x(t), \bar{x}(1))$ one can check

$$
\left.\frac{\partial^{3}}{\partial s \partial t^{2}}\right|_{(s, t)=\left(1, t_{0}\right)} H(t, \bar{x}(s))=\left.\frac{\partial^{3}}{\partial s \partial t^{2}}\right|_{(s, t)=\left(1, t_{0}\right)} c(x(t), \bar{x}(s))=0 .
$$

These last two facts together imply that $s \in[0,1] \mapsto f(s)=\frac{\partial^{2} H}{\partial t^{2}}\left(t_{0}, \bar{x}(s)\right)$ is a concave function attaining its maximum at $s=1$. The desired non-negativity $0 \leq f(0) \leq f(1)=$ $\frac{\partial^{2} H}{\partial t^{2}}\left(t_{0}, \bar{x}\right)$ then follows from A.5.

Use the same notation as in Claim A.2 Define $g(t, \bar{x})=\frac{\partial}{\partial t} H(t, \bar{x})$ and set

$$
S^{+}(t)=\{\bar{x} \in \bar{M} \mid g(t, \bar{x}) \geq 0\} .
$$

Notice $\bar{D} g(t, \bar{x}) \neq 0$ on $[0,1] \times \bar{M}$, so $\partial S^{+}(t)=\{\bar{x} \in \bar{M} \mid g(t, \bar{x})=0\}$. Extending the geodesic $(x(t), \bar{x}) \in N$ to values of $t$ slightly beyond [0,1], we can apply Lemma 4.9 Claim A.2 shows the set-valued function $S^{+}(t)$ is non-decreasing in $t$; that is, $S^{+}\left(t_{1}\right) \subset$ $S^{+}\left(t_{2}\right)$ for $0 \leq t_{1} \leq t_{2} \leq 1$. Let $\bar{x}_{0}=F\left(x_{0}\right)$. Then $g\left(0, \bar{x}_{0}\right)=0$, thus $\bar{x}_{0} \in S^{+}(0)$ and the monotonicity of $S^{+}(t)$ implies $\bar{x}_{0} \in S^{+}(t)$ for all $0 \leq t \leq 1$. Thus $g\left(t, \bar{x}_{0}\right) \geq 0$ and

$$
u(x)+c\left(x, \bar{x}_{0}\right)=H\left(1, \bar{x}_{0}\right) \geq H\left(0, \bar{x}_{0}\right)=u\left(x_{0}\right)+c\left(x_{0}, \bar{x}_{0}\right),
$$

completing the proof.

Remark A.3. Given $F: M \mapsto \bar{M}$, Lemma A.1 of [10] shows any Radon measure $\gamma \geq 0$ on $M \times \bar{M}$ assigning zero outer measure to the complement of $\operatorname{Graph}(F)$ has its first marginal $\rho:=\pi_{\#} \gamma$ pushed forward to its second marginal $\bar{\rho}:=\bar{\pi}_{\#} \gamma=F_{\#} \rho$ by the map $F$. Here $\pi(x, \bar{x})=x$ and $\bar{\pi}(x, \bar{x})=\bar{x}$. This applies in particular to the Riemannian volume $\gamma$ induced on a spacelike $\operatorname{submanifold} \operatorname{Graph}(F)$ by the pseudo-metric $h$. 
Acknowledgments. It is a pleasure to thank Neil Trudinger, Xu-Jia Wang, Grégoire Loeper, and Cédric Villani for many fruitful discussions, and for providing us with preprints of their inspiring works. Useful comments were also provided by John Bland, Robert Bryant, Philippe Delanoë, Stamatis Dostoglou, Nassif Ghoussoub, Gerhard Huisken, Tom Ilmanen, John Lott, Peter Michor, Truyen Nguyen, and Micah Warren. We thank Jeff Viaclovsky for helpful references, and Brendan Pass for a careful reading of the manuscript. We are grateful to the Mathematical Sciences Research Institute in Berkeley, where parts of this paper were written, and to Adrian Nachman, Jim Colliander, and the 2006-07 participants of Fields Analysis Working Group, for the stimulating environment which they helped to create. An extended abstract announcing the results contained herein appeared in [37].

This research was supported in part by Natural Sciences and Engineering Research Council of Canada Grant 217006-03 and United States National Science Foundation Grant DMS-0354729. This paper supersedes the earlier paper [28].

\section{References}

[1] Ambrosio, L. A., Gigli, N., Savaré, G.: Gradient Flows in Metric Spaces and in the Space of Probability Measures. Lecture Notes in Math. ETH Zürich, Birkhäuser, Basel (2005) Zbl 1090.35002 MR 2129498

[2] Besse, A.: Einstein Manifolds. Springer, Berlin (1987) Zbl 0613.53001 MR 0867684

[3] Brenier, Y.: Décomposition polaire et réarrangement monotone des champs de vecteurs. C. R. Acad. Sci. Paris Sér. I Math. 305, 805-808 (1987) Zbl 0652.26017 MR 0923203

[4] Caffarelli, L.: Allocation maps with general cost functions. In: P. Marcellini et al. (eds.), Partial Differential Equations and Applications, Lecture Notes in Pure Appl. Math. 177, Dekker, New York, 29-35 (1996) Zbl 0883.49030 MR 1371577

[5] Caffarelli, L. A.: The regularity of mappings with a convex potential. J. Amer. Math. Soc. 5, 99-104 (1992) Zbl 0753.35031 MR 1124980

[6] Caffarelli, L. A.: Boundary regularity of maps with convex potentials. Comm. Pure Appl. Math. 45, 1141-1151 (1992) Zbl 0778.35015 MR 1177479

[7] Caffarelli, L. A.: Boundary regularity of maps with convex potentials-II. Ann. of Math. (2) 144, 453-496 (1996) Zbl 0916.35016 MR 1426885

[8] Caffarelli, L. A., Gutiérrez, C., Huang, Q.: On the regularity of reflector antennas. Ann. of Math. (2) 167, 299-323 (2008) Zbl 1140.35009 MR 2373156

[9] Carlier, G.: Duality and existence for a class of mass transportation problems and economic applications. Adv. Math. Econom. 5, 1-21 (2003) Zbl pre02134650 MR 2160899

[10] Chiappori, P.-A., McCann, R. J., Nesheim, L.: Hedonic price equilibria, stable matching, and optimal transport: equivalence, topology, and uniqueness. Econom. Theory 42, 317-354 (2010) Zbl 1183.91056 MR 2564439

[11] Cordero-Erausquin, D.: Sur le transport de mesures périodiques. C. R. Acad. Sci. Paris Sér. I Math. 329, 199-202 (1999) Zbl 0942.28015 MR 1711060

[12] Cordero-Erausquin, D.: Non-smooth differential properties of optimal transport. In: Recent Advances in the Theory and Applications of Mass Transport, Contemp. Math. 353, Amer. Math. Soc., Providence, 61-71 (2004) Zbl 1141.49040 MR 2079070

[13] Delanoë, P.: Classical solvability in dimension two of the second boundary-value problem associated with the Monge-Ampère operator. Ann. Inst. H. Poincaré Anal. Non Linéaire 8, 443-457 (1991) Zbl 0778.35037||MR 1136351

[14] Delanoë, P.: Gradient rearrangement for diffeomorphisms of a compact manifold. Differential Geom. Appl. 20, 145-165 (2004) Zbl 1039.58008 MR 2038552 
[15] Delanoë, P., Ge, Y.: Regularity of optimal transportation maps on compact, locally nearly spherical, manifolds. J. Reine Angew. Math., to appear

[16] Delanoë, P., Loeper, G.: Gradient estimates for potentials of invertible gradient-mappings on the sphere. Calc. Var. Partial Differential Equations 26, 297-311 (2006) Zbl 1136.35358 MR 2232207

[17] Figalli, A., Kim, Y.-H., McCann, R. J.: Continuity and injectivity of optimal maps for nonnegatively cross-curved costs. arXiv:0911.3952v1

[18] Figalli, A., Loeper, G.: $C^{1}$ regularity of solutions of the Monge-Ampère equation for optimal transport in dimension two. Calc. Var. Partial Differential Equations 35, 537-550 (2009) Zbl 1170.35400 MR 2496656

[19] Figalli, A., Rifford, L.: Continuity of optimal transport maps and convexity of injectivity domains on small deformations of $\mathbb{S}^{2}$. Comm. Pure Appl. Math. 62, 1670-1706 (2009) Zbl 1170.35400 MR 2569074

[20] Figalli, A., Villani, C.: An approximation lemma about the cut locus, with applications in optimal transport theory. Methods Appl. Anal. 15, 149-154 (2008) Zbl 1175.49040 MR 2481676

[21] Gangbo, W.: Habilitation thesis. Université de Metz (1995)

[22] Gangbo, W., McCann, R. J.: The geometry of optimal transportation. Acta Math. 177, 113161 (1996) Zbl 0887.49017 MR 1440931

[23] Gangbo, W., McCann, R. J.: Shape recognition via Wasserstein distance. Quart. Appl. Math. 58, 705-737 (2000) Zbl 1039.49038 MR 1788425

[24] Glimm, T., Oliker, V.: Optical design of single reflector systems and the Monge-Kantorovich mass transfer problem. J. Math. Sci. 117, 4096-4108 (2003) Zbl 1049.49030 MR 2027449

[25] Kantorovich, L.: On the translocation of masses. C.R. (Doklady) Acad. Sci. URSS (N.S.) 37, 199-201 (1942) Zbl 0061.09705 MR 0009619

[26] Kim, Y.-H.: Counterexamples to continuity of optimal transportation on positively curved Riemannian manifolds. Int. Math. Res. Notices 2008, art ID rnn120, 15 pp. Zbl 1160.49047 MR 2448078

[27] Kim, Y.-H., McCann, R. J.: Appendices to original version of "Continuity, curvature, and the general covariance of optimal transportation". arXiv:math/0712.3077v1

[28] Kim, Y.-H., McCann, R. J.: On the cost-subdifferentials of cost-convex functions. arXiv:math/ $0706.1226 \mathrm{v} 1$

[29] Kim, Y.-H., McCann, R. J.: Towards the smoothness of optimal maps on Riemannian submersions and Riemannian products (of round spheres in particular). arXiv:0806.0351v1; J. Reine Angew. Math., to appear

[30] Levin, V.: Abstract cyclical monotonicity and Monge solutions for the general MongeKantorovich problem. Set-Valued Anal. 7, 7-32 (1999) Zbl 0934.54013 MR 1699061

[31] Loeper, G.: On the regularity of solutions of optimal transportation problems. Acta Math. 202, 241-283 (2009) MR 2506751

[32] Loeper, G.: On the regularity of solutions of optimal transportation problems. The sphere case and the reflector antenna. Arch. Ration. Mech. Anal., to appear

[33] Loeper, G., Villani, C.: Regularity of optimal transport in curved geometry: the nonfocal case. Duke Math. J. 151, 431-485 (2010) Zbl pre05688245

[34] Ma, X.-N., Trudinger, N., Wang, X.-J.: Regularity of potential functions of the optimal transportation problem. Arch. Ration. Mech. Anal. 177, 151-183 (2005) Zbl 1072.49035 MR 2188047

[35] McCann, R. J.: Existence and uniqueness of monotone measure-preserving maps. Duke Math. J. 80, 309-323 (1995) Zbl 0873.28009 MR 1369395 
[36] McCann, R. J.: Polar factorization of maps on Riemannian manifolds. Geom. Funct. Anal. 11, 589-608 (2001) Zbl 1011.58009 MR 1844080

[37] McCann, R. J.: Curvature and continuity of optimal transport (with Y.-H. Kim). Oberwolfach Rep. 4, 2035-2078 (2007)

[38] Monge, G.: Mémoire sur la théorie des déblais et de remblais. Histoire de l'Académie Royale des Sciences de Paris, avec les Mémoires de Mathématique et de Physique pour la même année, 666-704 (1781)

[39] O’Neill, B.: Semi-Riemannian Geometry (with applications to Relativity). Pure Appl. Math. 103. Academic Press, San Diego (1983) Zbl 0531.53051 MR 0719023

[40] Trudinger, N. S.: Recent developments in elliptic partial differential equations of MongeAmpère type. In: International Congress of Mathematicians (Madrid, 2006), Volume 3, Eur. Math. Soc., Zürich, 291-301 (2006) Zbl 1130.35058 MR 2275682

[41] Trudinger, N. S., Wang, X.-J.: On convexity notions in optimal transportation. Preprint

[42] Trudinger, N. S., Wang, X.-J.: On the second boundary value problem for Monge-Ampère type equations and optimal transportation. Ann. Scuola Norm. Sup. Pisa Cl. Sci. (5) 8, 143 174 (2009) Zbl 1182.35134 MR 2512204

[43] Trudinger, N. S., Wang, X.-J.: On strict convexity and continuous differentiability of potential functions in optimal transportation. Arch. Ration. Mech. Anal. 192, 403-418 (2009) Zbl pre05598874 MR 2505359

[44] Urbas, J.: On the second boundary value problem for equations of Monge-Ampère type. J. Reine Angew. Math. 487, 115-124 (1997) Zbl 0880.35031 MR 1454261

[45] Viaclovsky, J. A.: Conformal geometry, contact geometry, and the calculus of variations. Duke Math. J. 101, 283-316 (2000) Zbl 0990.53035 MR 1738176

[46] Viaclovsky, J. A.: Conformally invariant Monge-Ampère equations: global solutions. Trans. Amer. Math. Soc. 352, 4371-4379 (2000) Zbl 0951.35044 MR 1694380

[47] Viaclovsky, J. A.: Conformal geometry and fully nonlinear equations. In: Ph. A. Griffiths (ed.), Inspired by S. S. Chern, Nankai Tracts Math. 11, World Sci., 435-460 (2006) Zbl 1142.53030 MR 2313345

[48] Villani, C.: Stability of a 4th-order curvature condition arising in optimal transport theory. J. Funct. Anal. 255, 2683-2708 (2008) Zbl 1158.53036 MR 2473273

[49] Villani, C.: Topics in Optimal Transportation, Grad. Stud. Math. 58, Amer. Math. Soc., Providence (2003) Zbl 1106.90001 MR 1964483

[50] Villani, C.: Optimal Transport, Old and New. Grundlehren Math. Wiss. 338, Springer, Berlin (2009) Zbl 1156.53003 MR 2459454

[51] Wald, R. M.: General Relativity. Univ. of Chicago Press, Chicago (1984) Zbl 0549.53001 MR 0757180

[52] Wang, X.-J.: On the design of a reflector antenna. Inverse Problems 12, 351-375 (1996) Zbl 0858.35142 MR 1391544

[53] Wang, X.-J.: On the design of a reflector antenna II. Calc. Var. Partial Differential Equations 20, 329-341 (2004) Zbl 1065.78013 MR 2062947

[54] Warren, M.: Calibrations associated to Monge-Ampère equations. Trans. Amer. Math. Soc. 362, 3947-3962 (2010)

[55] Wolfson, J. G.: Minimal Lagrangian diffeomorphisms and the Monge-Ampère equation. J. Differential Geom. 46, 335-373 (1997) Zbl 0926.53032 MR 1484047 